\title{
Convergence Analysis of Multirate Fixed-Stress Split Iterative Schemes for Coupling Flow with Geomechanics
}

\author{
T. Almani ${ }^{1,3}$, K. Kumar ${ }^{2}$, A. Dogru ${ }^{3}$, G. Singh ${ }^{1}$, M. F. Wheeler ${ }^{1}$ \\ ${ }^{1}$ Center for Subsurface Modeling, ICES, UT-Austin, Austin, TX 78712, USA \\ ${ }^{2}$ Mathematics Institute, University of Bergen, Norway \\ ${ }^{3}$ Saudi Arabian Oil Company, Saudi Arabia \\ \{tameem,gurpreet,mfw\}@ices.utexas.edu, kundan.kumar@uib.no, \\ ali.dogru@aramco.com
}

July 22,2016

\section{Abstract}

We consider multirate iterative schemes for the Biot system modelling coupled flow and geomechanics in a poro-elastic medium. The multirate iterative coupling scheme exploits the different time scales for the mechanics and flow problems by taking multiple finer time steps for flow within one coarse mechanics time step. We adapt the fixed stress split algorithm that decouples the flow and mechanics equations for the multirate case and perform an iteration between the two problems until convergence. We provide a fully discrete scheme that uses Backward Euler time discretization and mixed spaces for flow and conformal Galerkin for mechanics. Our analysis is based on studying the equations satisfied by the difference of iterates and using Banach contraction argument to prove that the corresponding scheme is a fixed point contraction. The analysis provides the value of an adjustable coefficient used in the proposed iterative coupling algorithms. Furthermore, we show that the converged quantities satisfy the variational weak form for the coupled discrete system.

Keywords. poroelasticity; Biot system; fixed-stress split iterative coupling; multirate scheme; contraction mapping

\section{Introduction}

The accurate modeling of coupled fluid flow and mechanical interactions has received more attention and importance for both environmental and petroleum engineering applications. Accurate 
and reliable numerical methods for solving such problems are needed for the accurate modeling of multiscale and multiphysics phenomena such as reservoir deformation, surface subsidence, well stability, sand production, waste deposition, pore collapse, fault activation, hydraulic fracturing, $\mathrm{CO}_{2}$ sequestration, and hydrocarbon recovery [16], [24]. Traditionally, changes in mechanical deformations are visible to fluid flow through a pore compressibility factor, which is insufficient for stress sensitive, and structurally weak reservoirs. In fact, it is only through the coupling between flow and mechanics that reliable reservoir models can be obtained. In several of the applications listed above, the mechanics and flow equations have different characteristic time scales. Multirate schemes exploit the different time scales of these two equations and allow taking different time steps for each of these two problems. This is naturally achieved by decoupling the two equations.

There are typically three different coupling approaches employed in modeling fluid flow coupled with reservoir geomechanics. They are known as the fully implicit, the explicit or loose coupling, and the iterative coupling methods. The fully implicit approach solves reservoir multiphase flow and mechanics equations simultaneously and enjoys excellent stability properties [19] though it poses certain computational challenges for the linear solver. On the other hand, the loosely coupled approach is less accurate, only conditionally stable but, contrary to the implicit coupling scheme, has a lower computational cost. The iterative coupling approach lies in between the two extremes, and solves the two coupled subsystems iteratively by exchanging the values of the shared state variables in an iterative manner. The procedure is iterated at each time step until the solution is obtained with an acceptable tolerance $[9,22,24,35]$. The iterative coupling approach allows the use of existing reservoir simulators, is easy to implement, is robust and has fast convergence provided it has been designed appropriately. Our proposed numerical method is based on such an iterative approach. These iterative methods can be also used as a pre-conditioner for the fully implicit method. The work of Gai et al $[11,13]$ interpreted the fixed stress split iterative coupling scheme as an effective physics-based preconditioning strategy applied to a Richardson fixed point iteration. The same preconditioning operator can be applied to the fully implicit coupled system, enhancing the underlying Krylov subspace iteration as well $[7,8,11]$.

The coupled flow and geomechanics problem has been intensively investigated in the past. Starting from the pioneering work of Terzaghi [33] and Biot [5,6], several nonlinear extensions have been proposed and investigated [10,12,29]. The work of Settari and Mourits [28] proposed a robust iterative and explicit coupling schemes for coupling flow with geomechanics along with fracture propagation. The existence, uniqueness, and regularity of the Biot system have been investigated by a number of authors (Showalter [31], Phillips \& Wheeler [26], and Girault et al [15]). However, the development and analysis of theoretically convergent iterative coupling algorithms in poroelastic media have received relatively less attention. Recently, the work of Mikelić and Wheeler [25] establishes geometric convergence (contraction with respect to appropriately chosen metrics) for different flow and geomechanics iterative coupling schemes. In addition, Kim et al. $[18,19]$ have used von Neumann stability analysis to study the stability and convergence of similar schemes. Moreover, the stability of the multirate explicit coupling schemes has been recently investigated and established for the quasi-static Biot system under mild conditions on material parameters [2]. 
Our work is inspired from the previous work of Mikelić and Wheeler [25] (see also [14]) and extends their results to cover the case of fully discrete multirate iterative coupling schemes. Convergence properties of multirate explicit coupling schemes have been heavily investigated in [30,38] for the non-stationary Stokes-Darcy model. In contrast, we consider multirate iteratively coupled flow and geomechanics problems in this work. Figures 1.1a and 1.1b illustrate the differences between single rate versus multirate iterative coupling schemes. Figure 1.1a represents a typical single rate scheme, in which the flow and mechanics problems share the exact same time step, and the coupling iteration continues until convergence. In contrast, Figure 1.1b demonstrates a typical multirate scheme, in which the flow problem takes multiple finer local time steps within one coarser mechanics time step for each iterative coupling iteration. The process is iterated until convergence. In this work, we propose different multirate iterative schemes and their analyses and deduce the contracting character of each scheme. Convergence immediately follows by applying Banach's fixed point theorem. The presence of two different time steps for different equations in such a system of PDEs introduces several complications. We define an appropriate expression of the volumetric mean stress for the multirate scheme and use the flow and mechanics estimates to derive a contraction for the difference of two successive coupling iterates. In addition, we employ mathematical induction along with a compactness argument to deduce strong convergence of the pressure and flux unknowns for flow finer time steps within a coarser mechanics time step. Our analysis also reveals the optimal value of the fixed stress split regularization term in the mass conservation equation. Moreover, we introduce a modified multirate iterative coupling scheme that successively corrects the fluxes in even coupling iterations so that the resulting scheme has the same convergence properties as of single rate scheme. To the best of our knowledge, this is the first analysis of multirate schemes for Biot equations.

To summarize, our contributions in this work are as follows: we formulate two multirate iterative coupling schemes for the Biot system that can be viewed as the extensions of the classical fixedstress split coupling algorithm (see [25]) to the multirate settings in which flow takes finer time steps compared to the mechanics problem. Furthermore, we establish the contracting behavior of both schemes leading to geometric speed of convergence with an explicit expression for the contracting factor. In terms of numerical analysis, the novelty is in combining the contraction property with an induction argument to show that the obtained solution converges to the unique solution of the original weak formulation given in Definition 2.2. Moreover, the numerical examples show the sharpness of the theoretical estimates. This also reveals the CPU time savings as a result of the reduction in the number of mechanics linear iterations for the multirate scheme versus the single rate scheme. Finally, our proof outlines a general strategy that is likely to be useful for obtaining similar estimates in other contexts. For instance, a similar strategy has been applied to the multirate undrained-split coupling scheme in [3,4] improving the contraction obtained in [25].

The paper is structured as follows. We present the model and discretization in Section 2 . The multirate scheme is introduced and analysed in Section 3. We also present a modified multirate scheme along with its analysis in Section 4. Numerical results are shown in Section ??. We discuss the conclusions and outlook in Section 6. 


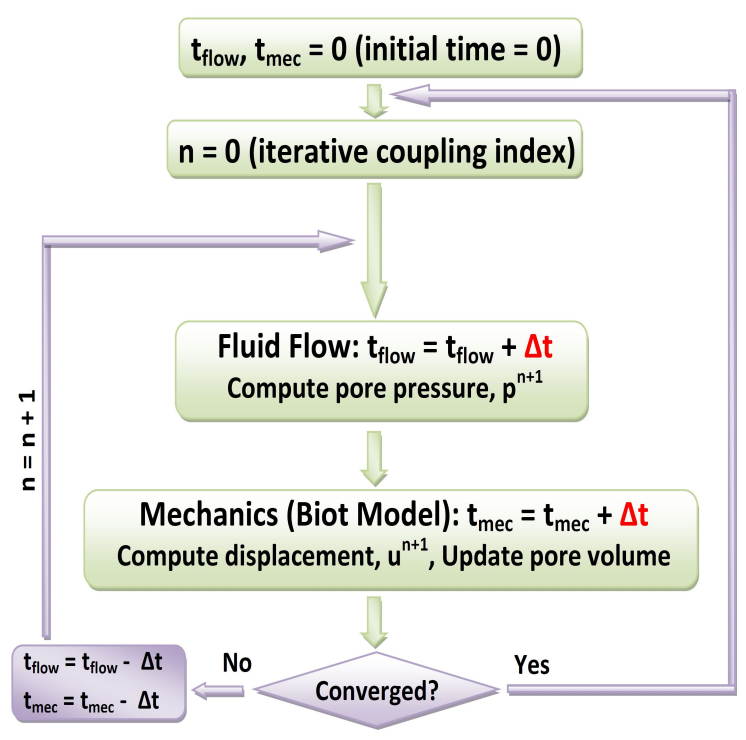

(a) Single Rate

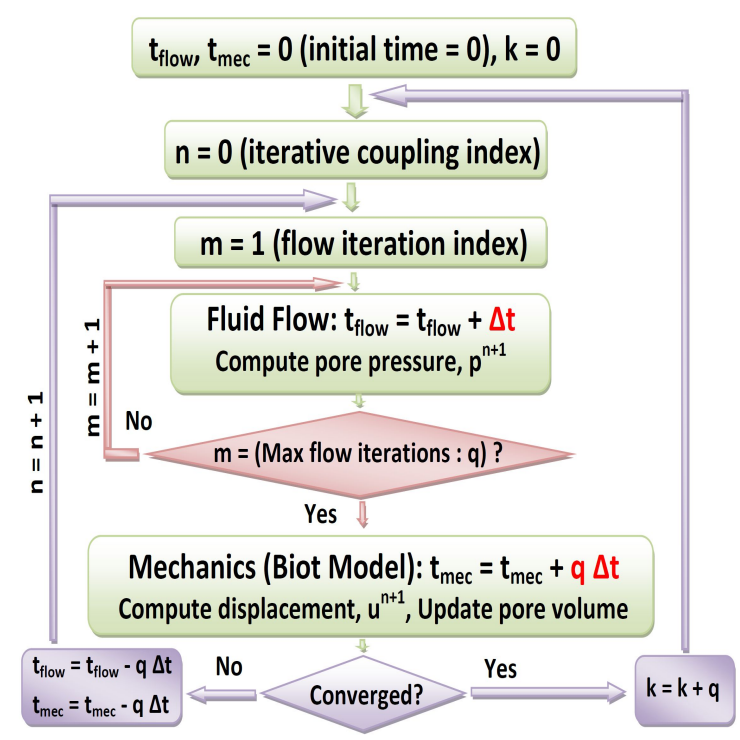

(b) Multirate

Figure 1.1: Flowchart for iterative algorithm using single rate and multirate time stepping for coupled geomechanics and flow problem

\subsection{Preliminaries}

Let $\Omega$ be a bounded domain (open and connected) of $\mathbb{R}^{d}$, where the dimension $d=2$ or 3 , with a Lipschitz continuous boundary $\partial \Omega$. For the pressure unknown, we assume that the boundary is decomposed into Dirichlet boundary $\Gamma_{D}$, and Neumann boundary $\Gamma_{N}$, associated with Dirichlet and Neumann boundary conditions respectively, such that $\Gamma_{D} \cup \Gamma_{N}=\partial \Omega$. In addition, Let $\mathfrak{D}(\Omega)$ be the space of all functions that are infinitely differentiable and with compact support in $\Omega$ and let $\mathfrak{D}^{\prime}(\Omega)$ be its dual space, i.e. the space of distributions in $\Omega$. As usual, we denote by $H^{1}(\Omega)$ the classical Sobolev space

$$
H^{1}(\Omega)=\left\{v \in L^{2}(\Omega) ; \nabla v \in L^{2}(\Omega)^{d}\right\}
$$

equipped with the semi-norm and norm:

$$
|v|_{H^{1}(\Omega)}=\|\nabla v\|_{L^{2}(\Omega)^{d}} \quad, \quad\|v\|_{H^{1}(\Omega)}=\left(\|v\|_{L^{2}(\Omega)}^{2}+|v|_{H^{1}(\Omega)}^{2}\right)^{1 / 2} .
$$

More generally, for $1 \leq p<\infty, W^{1, p}(\Omega)$ is the space

$$
W^{1, p}(\Omega)=\left\{v \in L^{p}(\Omega) ; \nabla v \in L^{p}(\Omega)^{d}\right\}
$$

normed by

$$
|v|_{W^{1, p}(\Omega)}=\|\nabla v\|_{L^{p}(\Omega)} \quad, \quad\|v\|_{W^{1, p}(\Omega)}=\left(\|v\|_{L^{p}(\Omega)}^{p}+|v|_{W^{1, p}(\Omega)}^{p}\right)^{1 / p}
$$


with the usual modification when $p=\infty$. We also define:

$$
H_{0}^{1}(\Omega)=\left\{v \in H^{1}(\Omega) ;\left.v\right|_{\partial \Omega}=0\right\},
$$

and for the divergence operator, we shall use the space

$$
H(\operatorname{div} ; \Omega)=\left\{\boldsymbol{v} \in L^{2}(\Omega)^{d} ; \nabla \cdot \boldsymbol{v} \in L^{2}(\Omega)\right\},
$$

equipped with the norm

$$
\|\boldsymbol{v}\|_{H(\mathrm{div} ; \Omega)}=\left(\|\boldsymbol{v}\|_{L^{2}(\Omega)}^{2}+\|\nabla \cdot \boldsymbol{v}\|_{L^{2}(\Omega)}^{2}\right)^{1 / 2} .
$$

We recall the definition of the symmetric strain tensor: $\varepsilon(v)=\frac{1}{2}\left(\nabla v+(\nabla v)^{T}\right)$, for a vector $v$ in $\mathbb{R}^{d}$.

\section{Model equations, discretization and splitting algorithm}

We assume a linear, elastic, homogeneous, and isotropic porous medium $\Omega \subset \mathbb{R}^{d}, d=2$ or 3 , in which the reservoir is saturated with a slightly compressible viscous fluid.

\subsection{Assumptions}

We have the following assumptions on the model and data:

1. For mechanical modeling, the reservoir is assumed to be homogeneous, isotropic and saturated poro-elastic medium. The reference density of the fluid $\rho_{f}>0$ is given and positive.

2. The Lamé coefficients $\lambda>0$ and $G>0$, the dimensionless Biot coefficient $\alpha$, and the pore volume $\varphi^{*}$ are all positive.

3. The fluid is assumed to be slightly compressible and its density is a linear function of pressure. The viscosity $\mu_{f}>0$ is assumed to be constant.

4. The absolute permeability tensor, $\boldsymbol{K}$, is assumed to be symmetric, bounded, uniformly positive definite in space and constant in time.

We will use here a quasi-static Biot model which is quite standard in literature [6,16]. The model reads: Find $\boldsymbol{u}$ and $p$ satisfying the equations below for all time $t \in] 0, T[$ : 


$$
\begin{gathered}
-\operatorname{div} \boldsymbol{\sigma}^{\mathrm{por}}(\boldsymbol{u}, p)=\boldsymbol{f} \text { in } \Omega, \\
\boldsymbol{\sigma}^{\mathrm{por}}(\boldsymbol{u}, p)=\boldsymbol{\sigma}(\boldsymbol{u})-\alpha p \boldsymbol{I} \text { in } \Omega, \\
\boldsymbol{\sigma}(\boldsymbol{u})=\lambda(\nabla \cdot \boldsymbol{u}) \boldsymbol{I}+2 G \boldsymbol{\varepsilon}(\boldsymbol{u}) \text { in } \Omega, \\
\frac{\partial}{\partial t}\left(\left(\frac{1}{M}+c_{f} \varphi_{0}\right) p+\alpha \nabla \cdot \boldsymbol{u}\right)-\nabla \cdot\left(\frac{1}{\mu_{f}} \boldsymbol{K}\left(\nabla p-\rho_{f, r} g \nabla \eta\right)\right)=\tilde{q} \text { in } \Omega,
\end{gathered}
$$

Boundary Conditions: $\boldsymbol{u}=\mathbf{0}$ on $\partial \Omega, \boldsymbol{K}\left(\nabla p-\rho_{f, r} g \nabla \eta\right) \cdot \boldsymbol{n}=0$ on $\Gamma_{N}, p=0$ on $\Gamma_{D}$,

Initial Condition $(t=0):\left(\left(\frac{1}{M}+c_{f} \varphi_{0}\right) p+\alpha \nabla \cdot \boldsymbol{u}\right)(0)=\left(\frac{1}{M}+c_{f} \varphi_{0}\right) p_{0}+\alpha \nabla \cdot \boldsymbol{u}_{0}$

where: $g$ is the gravitational constant, $\eta$ is the distance in the vertical direction (assumed to be constant in time), $\rho_{f, r}>0$ is a constant reference density (relative to the reference pressure $p_{r}$ ), $\varphi_{0}$ is the initial porosity, $M$ is the Biot modulus, $\tilde{q}=\frac{q}{\rho_{f, r}}$ where $q$ is a mass source or sink term taking into account injection into or out of the reservoir. We remark that the first three equations describe the mechanics whereas the fourth one is the flow equation. Note that the above system is linear and coupled.

\subsection{Mixed variational formulation}

We will use a mixed formulation for the flow and conformal Galerkin formulation for the mechanics equation. The mixed method defines flux as a separate unknown and rewrites the flow equation as a system of first order equations. Such a formulation for the flow is standard and is preferred because it is locally mass conservative and has an explicit computation for the flux. Accordingly, for the fully discrete formulation (discrete in time and space), we use a mixed finite element method for space discretization and a backward-Euler time discretization. Let $\mathfrak{T}_{h}$ denote a regular family of conforming triangular elements of the domain of interest, $\bar{\Omega}$. Using the lowest order RaviartThomas (RT) spaces, we have the following discrete spaces ( $\boldsymbol{V}_{h}$ for discrete displacements, $Q_{h}$ for discrete pressures, and $\boldsymbol{Z}_{h}$ for discrete velocities (fluxes)):

$$
\begin{aligned}
& \boldsymbol{V}_{h}=\left\{\boldsymbol{v}_{h} \in H^{1}(\Omega)^{d} ; \forall T \in \mathfrak{T}_{h}, \boldsymbol{v}_{h \mid T} \in \mathbb{P}_{1}^{d}, \boldsymbol{v}_{h \mid \partial \Omega}=\mathbf{0}\right\} \\
& Q_{h}=\left\{p_{h} \in L^{2}(\Omega) ; \forall T \in \mathfrak{T}_{h}, p_{h \mid T} \in \mathbb{P}_{0}\right\} \\
& \boldsymbol{Z}_{h}=\left\{\boldsymbol{q}_{h} \in H(\operatorname{div} ; \Omega) ; \forall T \in \mathfrak{T}_{h}, \boldsymbol{q}_{h \mid T} \in \mathbb{P}_{1}^{d}, \boldsymbol{q}_{h} \cdot \boldsymbol{n}=0 \text { on } \Gamma_{N}\right\}
\end{aligned}
$$

The space of displacements, $\boldsymbol{V}_{h}$, is equipped with the norm:

$$
\|\boldsymbol{v}\|_{V_{h}}=\left(\sum_{i=1}^{d}\left\|v_{i}\right\|_{\Omega}^{2}\right)^{1 / 2}
$$


We also assume that the finer time step is given by: $\Delta t_{k}=t_{k}-t_{k-1}$. In this work, we assume uniform fine flow time steps, so for simplicity, we will drop the subscript $k$, and denote the fine time step by $\Delta t$. If we denote the total number of timesteps by $\mathrm{N}$, then the total simulation time is given by $\mathrm{T}=\Delta t \mathrm{~N}$, and $t_{i}=i \Delta t, 0 \leqslant i \leqslant N$ denote the discrete time points.

For the fully discrete scheme, we have chosen the Raviart-Thomas spaces for the mixed finite element discretization. However, the proof extends to other choices for the mixed spaces and we will state the results for Multipoint Flux Mixed Finite Element (MFMFE) spaces [35,36] in Remark 4.4 .

Remark 2.1 Notation: In the following, there will be two indices, one for the time step and the other for the coupling between the flow and mechanics. To avoid any confusion, let us emphasise the following notations, $n$ denotes the coupling iteration index, $k$ is the coarser time step iteration index (for indexing mechanics coarse time steps), $m$ is the finer (local) time step iteration index (for indexing flow fine time steps), $\Delta t$ stands for the time step, and $q$ is the "fixed" number of local flow time steps per coarse mechanics time step. A schematic showing the relations between $k, m, q$, and $\Delta t$ can be found in figure $1.1 b$.

\subsection{Fully discrete scheme for multirate}

As discussed above, using the mixed finite element method in space and the backward Euler finite difference method in time, the weak formulation of a multirate scheme reads as follows.

Definition 2.2 For $k=i q, i \in \mathbb{N}$, and $1 \leq m \leq q$, find $p_{h}^{k+m} \in Q_{h}$, and $\boldsymbol{z}_{h}^{k+m} \in \boldsymbol{Z}_{h}$ such that, (flow equation)

$$
\begin{aligned}
\forall \theta_{h} \in Q_{h}, & \frac{1}{\Delta t}\left(\left(\frac{1}{M}+c_{f} \varphi_{0}\right)\left(p_{h}^{k+m}-p_{h}^{k+m-1}\right), \theta_{h}\right)+\frac{1}{\mu_{f}}\left(\nabla \cdot \boldsymbol{z}_{h}^{k+m}, \theta_{h}\right)= \\
& -\frac{\alpha}{q \Delta t}\left(\nabla \cdot\left(\boldsymbol{u}_{h}^{k+q}-\boldsymbol{u}_{h}^{k}\right), \theta_{h}\right)+\left(\tilde{q}_{h}, \theta_{h}\right), \\
\forall \boldsymbol{q}_{h} \in \boldsymbol{Z}_{h}, & \left(\boldsymbol{K}^{-1} \boldsymbol{z}_{h}^{k+m}, \boldsymbol{q}_{h}\right)=\left(p_{h}^{k+m}, \nabla \cdot \boldsymbol{q}_{h}\right)+\left(\rho_{f, r} g \nabla \eta, \boldsymbol{q}_{h}\right),
\end{aligned}
$$

and (mechanics equation)

find $\boldsymbol{u}_{h}^{k+q} \in \boldsymbol{V}_{h}$ such that,

$$
\forall \boldsymbol{v}_{h} \in V_{h}, 2 G\left(\boldsymbol{\varepsilon}\left(\boldsymbol{u}_{h}^{k+q}\right), \boldsymbol{\varepsilon}\left(\boldsymbol{v}_{h}\right)\right)+\lambda\left(\nabla \cdot \boldsymbol{u}_{h}^{k+q}, \nabla \cdot \boldsymbol{v}_{h}\right)-\alpha\left(p_{h}^{k+q}, \nabla \cdot \boldsymbol{v}_{h}\right)=\left(\boldsymbol{f}, \boldsymbol{v}_{h}\right) .
$$

with the initial condition for the first discrete time step,

$$
p_{h}^{0}=p_{0}
$$


Note that the pressure unknowns $p_{h}$ and flux unknowns $\boldsymbol{z}_{h}$ are being solved at finer time steps $t_{k+m}, m=0, \ldots, q$ whereas the mechanics variables $\boldsymbol{u}_{h}$ are being solved at $t_{i q}, i \in \mathbb{N}$. Therefore, for each mechanics time step of size $q \Delta t$, there are $q$ flow solves justifying the nomenclature of multirate. Moreover, the above system of PDEs is linear but coupled with the coupling terms being computed at the coarse time steps. Instead of solving the problem in a coupled manner, as discussed before, we will apply a splitting algorithm to decouple the two equations and iterate between them until the solutions satisfying the above system $(2.4)-(2.7)$ are obtained. In practice, there are 4 major splitting algorithms (drained, undrained, fixed strain and fixed stress) used for studying the Biot system depending upon whether one solves the mechanics first or flow and the physical variables which are being lagged. We will use the fixed stress splitting algorithm here because of its well established stability and excellent convergence properties as shown in Mikelić and Wheeler [25], and Mikelić et al. [24].

\subsection{Standard Fixed stress split algorithm}

In the fixed stress split iterative coupling algorithm, we first solve the flow problem followed by the geomechanics problem. Even though we use the splitting strategy at the discrete level, it is probably easier to see this in the continuous strong form. Recalling that $n$ denotes the coupling iteration index between the flow and mechanics problems, the steps are as follows:

Step (a): Given $\boldsymbol{u}^{n}$, we solve for $p^{n+1}, \boldsymbol{z}^{n+1}$

$$
\begin{gathered}
\left(\frac{1}{M}+c_{f} \varphi_{0}+\frac{\alpha^{2}}{\lambda}\right) \frac{\partial}{\partial t} p^{n+1}-\nabla \cdot \boldsymbol{z}^{n+1}=\frac{\alpha^{2}}{\lambda} \frac{\partial}{\partial t} p^{n}-\alpha \nabla \cdot \frac{\partial}{\partial t} \boldsymbol{u}^{n}+\tilde{q} \\
\boldsymbol{z}^{n+1}=\frac{1}{\mu_{f}} \boldsymbol{K}\left(\nabla p^{n+1}-\rho_{f, r} g \nabla \eta\right)
\end{gathered}
$$

Once the flow is computed, we update the displacement solution.

Step (b): Given $p^{n+1}, \boldsymbol{z}^{n+1}$, we solve for $\boldsymbol{u}^{n+1}$ satisfying

$$
\begin{aligned}
& -\operatorname{div} \boldsymbol{\sigma}^{\text {por }}\left(\boldsymbol{u}^{n+1}, p^{n+1}\right)=\boldsymbol{f} \\
& \boldsymbol{\sigma}^{\text {por }}\left(\boldsymbol{u}^{n+1}, p^{n+1}\right)=\boldsymbol{\sigma}\left(\boldsymbol{u}^{n+1}\right)-\alpha p^{n+1} \\
& \boldsymbol{\sigma}\left(\boldsymbol{u}^{n+1}\right)=\lambda\left(\nabla \cdot \boldsymbol{u}^{n+1}\right) \boldsymbol{I}+2 G \boldsymbol{\varepsilon}\left(\boldsymbol{u}^{n+1}\right)
\end{aligned}
$$

with the initial condition, independent of n,

$$
\left(\left(\frac{1}{M}+c_{f} \varphi_{0}\right) p^{n+1}+\alpha \nabla \cdot \boldsymbol{u}^{n+1}\right)(0)=\left(\frac{1}{M}+c_{f} \varphi_{0}\right) p_{0}+\alpha \nabla \cdot \boldsymbol{u}_{0}
$$

Note that the flow equation has a regularization term $\alpha^{2} / \lambda \partial_{t} p^{n+1}$ added to the left hand side and a similar term added to the right hand side for consistence while the mechanics equation remains unchanged. In the case of convergence, this term vanishes retrieving the original equation. Indeed, this has been analyzed in literature and we simply state the results to elucidate our approach. Following result is obtained in Mikelić and Wheeler [25], and adapted to our model equations. 
Theorem 2.3 [Mikelić \& Wheeler [25]] Let $\Omega^{t}:=\Omega \times(0, t), \sigma_{v}:=\sigma_{v, 0}+\lambda \nabla \cdot \boldsymbol{u}-\alpha p,\left.\sigma_{v}\right|_{t=0}=\sigma_{v, 0}$ (the initial volumetric mean total stress), $\sigma_{v}^{n}:=\sigma_{v, 0}+\lambda \nabla \cdot \boldsymbol{u}^{n}-\alpha p^{n}$, and $\delta$ denoting the difference of two successive iterates, the fixed stress split scheme as given in Section 2.4 is a contraction given by

$$
\begin{gathered}
\left\|\partial_{t} \delta \sigma_{v}^{n+1}\right\|_{\Omega^{t}}^{2}+\frac{\lambda \alpha^{2}}{\mu_{f}\left(M \alpha^{2}+\lambda\left(1+M c_{f} \varphi_{0}\right)\right)}\left\|K^{-1 / 2} \delta \nabla p^{n+1}(t)\right\|_{\Omega}^{2}+4 G \lambda\left\|\varepsilon\left(\partial_{t} \delta \boldsymbol{u}^{n+1}\right)\right\|_{\Omega^{t}}^{2}+\lambda^{2}\left\|\nabla \cdot \delta \boldsymbol{u}^{n+1, k}\right\|_{\Omega^{t}}^{2} \\
\leq\left(\frac{M \alpha^{2}}{\lambda+M \lambda c_{f} \varphi_{0}+M \alpha^{2}}\right)^{2}\left\|\partial_{t} \delta \sigma_{v}^{n}\right\|_{\Omega^{t}}^{2}
\end{gathered}
$$

The proof of the above results can be adapted to the fully discrete case in which a mixed formulation is used for space discretization (see section 4 and Theorem 4.3). Moreover, in the Theorem 2.3 , the contraction is obtained on the volumetric mean stress, $\sigma_{v}$, involving both pressure (flow) and displacement (mechanics) unknowns. A relatively straightforward argument shows that the converged quantities solve the original coupled equations in a weak form.

In what follows, we will derive similar estimates for the case of the multirate iterative coupling scheme. Two different multirate iterative coupling algorithms are discussed and analyzed below. Even though our approach is similar to the one in [25], the fact that we solve for multiple flow finer time steps within one coarser mechanics time step leads to several complications. The adaptation of the fixed stress algorithm requires defining an appropriate mean stress quantity and the analysis introduces two adjustable parameters. Careful algebraic manipulations are required to show the contraction. Even after the contraction is achieved, the presence of the two different time scales in the coupled problem requires non-trivial arguments involving the mathematical induction to show convergence to the weak formulation $(2.4)-(2.7)$.

\section{Multirate iterative coupling schemes}

\subsection{Multirate iterative scheme}

Here, we provide a multirate formulation of the "fixed stress split" iterative coupling algorithm and analyze its convergence properties in the next section. Recall that $n$ denotes the coupling iteration index, $k$ the coarser time step iteration index (for indexing mechanics time steps), $m$ the finer (local) time step iteration index (for indexing flow finer time steps), $\Delta t$ the unit time step, and $q$ denote "fixed" number of local flow time steps within one coarse mechanics time step. We begin by describing the algorithm. 


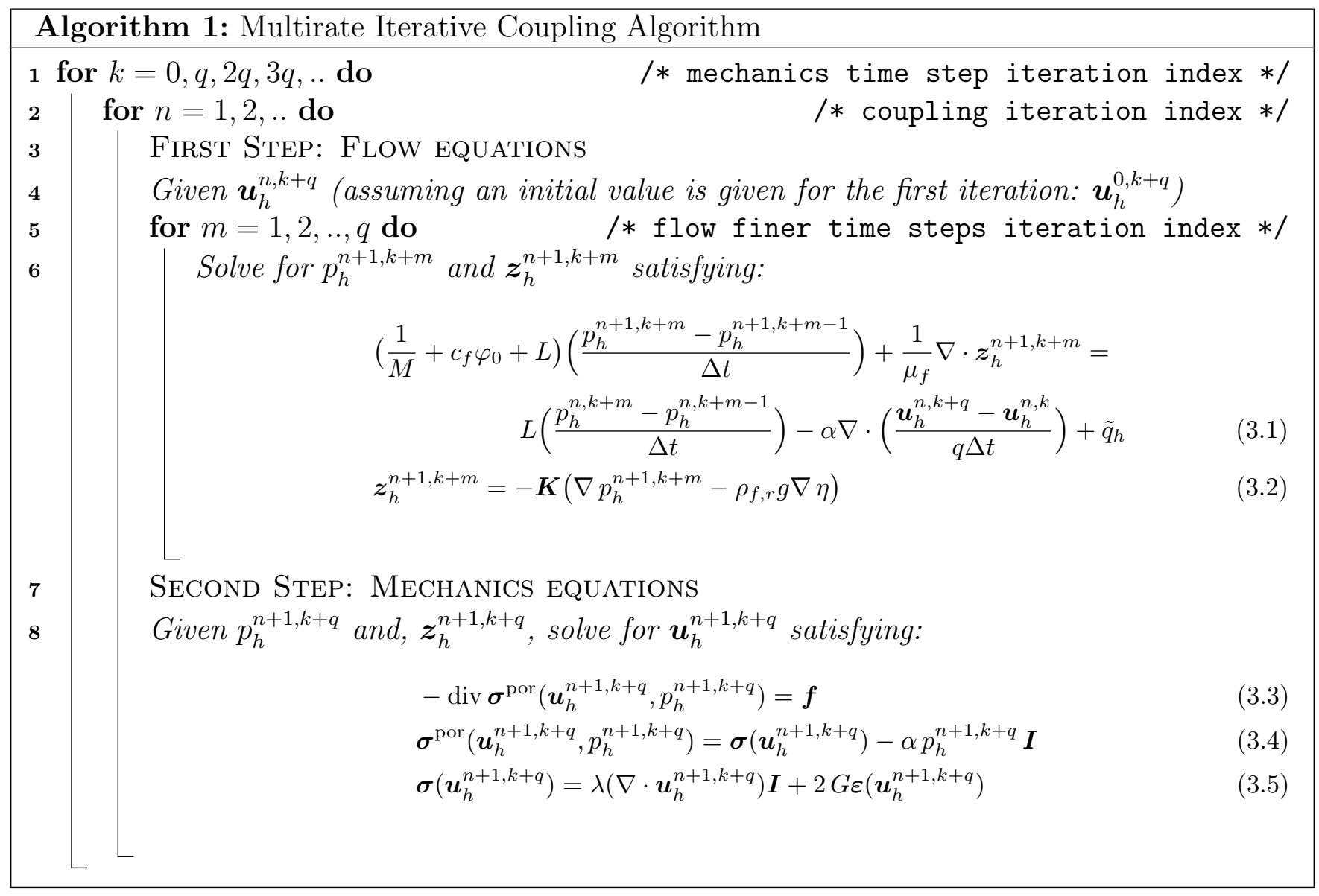


The weak formulation of equations (3.1) - (3.5) reads:

For $k=i q, i \in \mathbb{N}, n=1,2, .$.

- Step (a) Given $\boldsymbol{u}_{h}^{n, k+q}$ (with a specified initial value, $\boldsymbol{u}_{h}^{0, k+q}$, for the first iterative coupling iteration), for $1 \leq m \leq q$, find $p_{h}^{n+1, k+m} \in Q_{h}$, and $\boldsymbol{z}_{h}^{n+1, k+m} \in \boldsymbol{Z}_{h}$ such that,

$$
\begin{gathered}
\forall \theta_{h} \in Q_{h}, \frac{1}{\Delta t}\left(\left(\frac{1}{M}+c_{f} \varphi_{0}+L\right)\left(p_{h}^{n+1, k+m}-p_{h}^{n+1, k+m-1}\right), \theta_{h}\right)+\frac{1}{\mu_{f}}\left(\nabla \cdot \boldsymbol{z}_{h}^{n+1, k+m}, \theta_{h}\right)= \\
\frac{1}{\Delta t}\left(L\left(p_{h}^{n, k+m}-p_{h}^{n, k+m-1}\right)-\frac{\alpha}{q} \nabla \cdot\left(\boldsymbol{u}_{h}^{n, k+q}-\boldsymbol{u}_{h}^{n, k}\right), \theta_{h}\right)+\left(\tilde{q}_{h}, \theta_{h}\right) \\
\forall \boldsymbol{q}_{h} \in \boldsymbol{Z}_{h},\left(\boldsymbol{K}^{-1} \boldsymbol{z}_{h}^{n+1, k+m}, \boldsymbol{q}_{h}\right)=\left(p_{h}^{n+1, k+m}, \nabla \cdot \boldsymbol{q}_{h}\right)+\left(\rho_{f, r} g \nabla \eta, \boldsymbol{q}_{h}\right)
\end{gathered}
$$

with the initial condition, independent of $n$, for the first discrete time step,

$$
p_{h}^{n+1,0}=p_{0} .
$$

- Step (b) Given $p_{h}^{n+1, k+q}$ and, $\boldsymbol{z}_{h}^{n+1, k+q}$, find $\boldsymbol{u}_{h}^{n+1, k+q} \in \boldsymbol{V}_{h}$ such that,

$$
\forall \boldsymbol{v}_{h} \in V_{h}, 2 G\left(\boldsymbol{\varepsilon}\left(\boldsymbol{u}_{h}^{n+1, k+q}\right), \boldsymbol{\varepsilon}\left(\boldsymbol{v}_{h}\right)\right)+\lambda\left(\nabla \cdot \boldsymbol{u}_{h}^{n+1, k+q}, \nabla \cdot \boldsymbol{v}_{h}\right)-\alpha\left(p_{h}^{n+1, k+q}, \nabla \cdot \boldsymbol{v}_{h}\right)=\left(\boldsymbol{f}, \boldsymbol{v}_{h}\right) .
$$

In the above scheme, $L$ is the adjustable coefficient that will be chosen appropriately later (this choice completely determines the scheme) and $q$ is a user-defined number of finer flow steps. Below we analyze the above weak formulation and deduce the contracting character of the iterative scheme. The proof relies on studying the difference of two successive iterates and uses Banach's fixed point theorem. The final step is to show that the converged quantities satisfy the weak formulation (2.4) $-(2.7)$.

\subsection{Proof of contraction}

For a given time step $t=t_{k}$, we define the difference between two coupling iterates as:

$$
\delta \xi^{n+1, k}=\xi^{n+1, k}-\xi^{n, k}
$$

where $\xi$ may stand for $p_{h}, \boldsymbol{z}_{h}$, and $\boldsymbol{u}_{h}$. In addition, for notational convenience, we define,

$$
\beta=\frac{1}{M}+c_{f} \varphi_{0}+L
$$




\section{- Step 1: Flow equations}

For $n \geq 1$, by taking the difference of two successive iterates of (3.6), which corresponds to one local flow iteration and its corresponding local flow iteration in the previous flow and geomechanics iterative coupling iteration, testing with $\theta_{h}=\delta p_{h}^{n+1, k+m}-\delta p_{h}^{n+1, k+m-1}$, we obtain

$$
\begin{gathered}
\beta\left\|\delta p_{h}^{n+1, k+m}-\delta p_{h}^{n+1, k+m-1}\right\|^{2}+\frac{\Delta t}{\mu_{f}}\left(\nabla \cdot \delta \boldsymbol{z}_{h}^{n+1, k+m}, \delta p_{h}^{n+1, k+m}-\delta p_{h}^{n+1, k+m-1}\right)= \\
\left(L\left(\delta p_{h}^{n, k+m}-\delta p_{h}^{n, k+m-1}\right)-\frac{\alpha}{q}\left(\nabla \cdot \delta \boldsymbol{u}_{h}^{n, k+q}-\nabla \cdot \delta \boldsymbol{u}_{h}^{n, k}\right), \delta p_{h}^{n+1, k+m}-\delta p_{h}^{n+1, k+m-1}\right) .
\end{gathered}
$$

Similarly, for the flux equation (3.7), by taking the difference of two successive iterates, followed by taking the difference at two consecutive finer time steps, $t=t_{k+m}$, and $t=t_{k+m-1}$, and testing with $\boldsymbol{q}_{h}=\delta \boldsymbol{z}_{h}^{n+1, k+m}$, we obtain

$$
\left(\boldsymbol{K}^{-1}\left(\delta \boldsymbol{z}_{h}^{n+1, k+m}-\delta \boldsymbol{z}_{h}^{n+1, k+m-1}\right), \delta \boldsymbol{z}_{h}^{n+1, k+m}\right)=\left(\delta p_{h}^{n+1, k+m}-\delta p_{h}^{n+1, k+m-1}, \nabla \cdot \delta \boldsymbol{z}_{h}^{n+1, k+m}\right) .
$$

We combine (3.11) with (3.12), apply Young's inequality and use $\nabla \cdot \delta \boldsymbol{u}_{h}^{n, k}=0$ to obtain

$$
\begin{gathered}
\beta\left\|\delta p_{h}^{n+1, k+m}-\delta p_{h}^{n+1, k+m-1}\right\|^{2}+\frac{\Delta t}{\mu_{f}}\left(\boldsymbol{K}^{-1}\left(\delta \boldsymbol{z}_{h}^{n+1, k+m}-\delta \boldsymbol{z}_{h}^{n+1, k+m-1}\right), \delta \boldsymbol{z}_{h}^{n+1, k+m}\right) \leq \\
\frac{1}{2 \epsilon}\left\|L\left(\delta p_{h}^{n, k+m}-\delta p_{h}^{n, k+m-1}\right)-\frac{\alpha}{q} \nabla \cdot \delta \boldsymbol{u}_{h}^{n, k+q}\right\|^{2}+\frac{\epsilon}{2}\left\|\delta p_{h}^{n+1, k+m}-\delta p_{h}^{n+1, k+m-1}\right\|^{2} \cdot
\end{gathered}
$$

The choice $\epsilon=\beta$ absorbs the pressure term on the right hand side. Together with a simple expansion of the flux product, we derive

$$
\begin{aligned}
& \frac{\beta}{2}\left\|\delta p_{h}^{n+1, k+m}-\delta p_{h}^{n+1, k+m-1}\right\|^{2}+\frac{\Delta t}{2 \mu_{f}}\left\{\left\|\boldsymbol{K}^{-1 / 2} \delta \boldsymbol{z}_{h}^{n+1, k+m}\right\|^{2}-\left\|\boldsymbol{K}^{-1 / 2} \delta \boldsymbol{z}_{h}^{n+1, k+m-1}\right\|^{2}\right. \\
& \left.\quad+\left\|\boldsymbol{K}^{-1 / 2}\left(\delta \boldsymbol{z}_{h}^{n+1, k+m}-\delta \boldsymbol{z}_{h}^{n+1, k+m-1}\right)\right\|^{2}\right\} \leq \frac{1}{2 \beta}\left\|L\left(\delta p_{h}^{n, k+m}-\delta p_{h}^{n, k+m-1}\right)-\frac{\alpha}{q} \nabla \cdot \delta \boldsymbol{u}_{h}^{n, k+q}\right\|^{2} .
\end{aligned}
$$

The right hand side constitutes an expression for a quantity to be contracted on. Introducing a new parameter $\chi$, we define the volumetric mean stress for $(1 \leq m \leq q)$ as

$$
\chi \delta \sigma_{v}^{n, k+m}=L\left(\delta p_{h}^{n, k+m}-\delta p_{h}^{n, k+m-1}\right)-\frac{\alpha}{q} \nabla \cdot \delta \boldsymbol{u}_{h}^{n, k+q} .
$$

The value of $\chi$ will be chosen such that contraction can be achieved on the norm of $\sigma_{v}^{n, k+m}$, summed over q flow finer time steps, within one coarser mechanics time step. Multiplying 
(3.13) by $\frac{2}{\beta}$, summing up for $1 \leq m \leq q$, substituting the new definition of the volumetric mean stress (3.14), and noting that $\delta \boldsymbol{z}_{h}^{n+1, k}=0$, we obtain

$$
\begin{aligned}
& \sum_{m=1}^{q}\left\|\delta p_{h}^{n+1, k+m}-\delta p_{h}^{n+1, k+m-1}\right\|^{2}+\frac{\Delta t}{\beta \mu_{f}}\left\|\boldsymbol{K}^{-1 / 2} \delta \boldsymbol{z}_{h}^{n+1, k+q}\right\|^{2} \\
&+\frac{\Delta t}{\beta \mu_{f}} \sum_{m=1}^{q}\left\|\boldsymbol{K}^{-1 / 2}\left(\delta \boldsymbol{z}_{h}^{n+1, k+m}-\delta \boldsymbol{z}_{h}^{n+1, k+m-1}\right)\right\|^{2} \leq \frac{1}{\beta^{2}} \sum_{m=1}^{q}\left\|\chi \delta \sigma_{v}^{n, k+m}\right\|^{2} .
\end{aligned}
$$

\section{- Step 2: Elasticity equation}

For $n \geq 1$, we take the difference of successive iterates of the mechanics equation (3.9), multiply by a newly introduced parameter, $c_{0}$, and test with $\boldsymbol{v}_{h}=\delta \boldsymbol{u}_{h}^{n+1, k+q}$ to get

$$
2 G c_{0}\left\|\boldsymbol{\varepsilon}\left(\delta \boldsymbol{u}_{h}^{n+1, k+q}\right)\right\|^{2}+\lambda c_{0}\left\|\nabla \cdot \delta \boldsymbol{u}_{h}^{n+1, k+q}\right\|^{2}-\alpha c_{0}\left(\delta p_{h}^{n+1, k+q}, \nabla \cdot \delta \boldsymbol{u}_{h}^{n+1, k+q}\right)=0 .
$$

For the iterative scheme to be contractive, a quantity similar to the right hand side of (3.15), for the next iterative coupling iteration, $n+1$, has to be formed. To achieve that, we introduce a term involving a summation over all flow finer time steps in (3.16) by noticing that

$$
\sum_{m=1}^{q}\left(\delta p_{h}^{n+1, k+m}-\delta p_{h}^{n+1, k+m-1}\right)=\delta p_{h}^{n+1, k+q}
$$

Substituting (3.17) into (3.16) leads to

$$
\begin{aligned}
2 G c_{0}\left\|\boldsymbol{\varepsilon}\left(\delta \boldsymbol{u}_{h}^{n+1, k+q}\right)\right\|^{2}+\lambda c_{0} \| & \nabla \cdot \delta \boldsymbol{u}_{h}^{n+1, k+q} \|^{2} \\
& -\alpha c_{0}\left(\sum_{m=1}^{q}\left(\delta p_{h}^{n+1, k+m}-\delta p_{h}^{n+1, k+m-1}\right), \nabla \cdot \delta \boldsymbol{u}_{h}^{n+1, k+q}\right)=0 .
\end{aligned}
$$

\section{- Step 3: Combining flow and elasticity equations}

By combining (3.18) with (3.15), and rearranging terms, we form a square term, in expanded form, summed over flow finer time steps within one coarser mechanics time step,

$$
\begin{aligned}
& 2 G c_{0}\left\|\boldsymbol{\varepsilon}\left(\delta \boldsymbol{u}_{h}^{n+1, k+q}\right)\right\|^{2}+\sum_{m=1}^{q}\left\{\left\|\delta p_{h}^{n+1, k+m}-\delta p_{h}^{n+1, k+m-1}\right\|^{2}+\frac{\lambda c_{0}}{q}\left\|\nabla \cdot \delta \boldsymbol{u}_{h}^{n+1, k+q}\right\|^{2}\right. \\
& \left.-\alpha c_{0}\left(\delta p_{h}^{n+1, k+m}-\delta p_{h}^{n+1, k+m-1}, \nabla \cdot \delta \boldsymbol{u}_{h}^{n+1, k+q}\right)\right\}+\frac{\Delta t}{\beta \mu_{f}}\left\|\boldsymbol{K}^{-1 / 2} \delta \boldsymbol{z}_{h}^{n+1, k+q}\right\|^{2} \\
& +\frac{\Delta t}{\beta \mu_{f}} \sum_{m=1}^{q}\left\|\boldsymbol{K}^{-1 / 2}\left(\delta \boldsymbol{z}_{h}^{n+1, k+m}-\delta \boldsymbol{z}_{h}^{n+1, k+m-1}\right)\right\|^{2} \leq \frac{\chi^{2}}{\beta^{2}} \sum_{m=1}^{q}\left\|\delta \sigma_{v}^{n, k+m}\right\|^{2}
\end{aligned}
$$


It remains to choose the values of our newly introduced parameters, $\chi, L$, and $c_{0}$, such that the coefficients of the expanded square contributes only positive terms to the left hand side of (3.19). Therefore, we expand the right hand side of (3.19) as

$$
\begin{aligned}
\left\|\delta \sigma_{v}^{n, k+m}\right\|^{2} & =\frac{L^{2}}{\chi^{2}}\left\|\delta p_{h}^{n, k+m}-\delta p_{h}^{n, k+m-1}\right\|^{2}-\frac{2 \alpha L}{q \chi^{2}}\left(\delta p_{h}^{n, k+m}-\delta p_{h}^{n, k+m-1}, \nabla \cdot \delta \boldsymbol{u}_{h}^{n, k+q}\right) \\
& +\frac{\alpha^{2}}{\chi^{2} q^{2}}\left\|\nabla \cdot \delta \boldsymbol{u}_{h}^{n, k+q}\right\|^{2}
\end{aligned}
$$

Now, we match the coefficients of the expansion in (3.20) to the coefficients of the expanded square on the right hand side of (3.19). For the left hand side of (3.19) to remain positive, the following inequalities should be satisfied

$$
1 \geq \frac{L^{2}}{\chi^{2}}, \quad \frac{2 \alpha L}{q \chi^{2}}=\alpha c_{0}, \quad \frac{\lambda c_{0}}{q} \geq \frac{\alpha^{2}}{\chi^{2} q^{2}} .
$$

The second inequality gives rise to $c_{0}=\frac{2 L}{q \chi^{2}}$. The third inequality gives $L \geq \frac{\alpha^{2}}{2 \lambda}$. Since the contraction factor is monotone with respect to $\mathrm{L}$, its minimum is achieved when $L=\frac{\alpha^{2}}{2 \lambda}$. The first inequality gives $\chi^{2} \geq L^{2}$. The minimum value of the contraction factor is achieved when $\chi^{2}=L^{2}$. Therefore, with

$$
L=\frac{\alpha^{2}}{2 \lambda}, \quad \chi^{2}=L^{2}, \quad c_{0}=\frac{2 L}{q \chi^{2}},
$$

we group the terms of the expanded square on the left hand side of (3.19) to form the quantity of contraction for the next iterative coupling iteration, $n+1$, as

$$
\begin{aligned}
& 2 G c_{0}\left\|\boldsymbol{\varepsilon}\left(\delta \boldsymbol{u}_{h}^{n+1, k+q}\right)\right\|^{2}+\sum_{m=1}^{q}\left\|\delta \sigma_{v}^{n+1, k+m}\right\|^{2}+\frac{\Delta t}{\beta \mu_{f}}\left\|\boldsymbol{K}^{-1 / 2} \delta \boldsymbol{z}_{h}^{n+1, k+q}\right\|^{2} \\
& +\frac{\Delta t}{\beta \mu_{f}} \sum_{m=1}^{q}\left\|\boldsymbol{K}^{-1 / 2}\left(\delta \boldsymbol{z}_{h}^{n+1, k+m}-\delta \boldsymbol{z}_{h}^{n+1, k+m-1}\right)\right\|^{2} \leq\left(\frac{L}{\frac{1}{M}+c_{f} \varphi_{0}+L}\right)^{2} \sum_{m=1}^{q}\left\|\delta \sigma_{v}^{n, k+m}\right\|^{2} .
\end{aligned}
$$

Clearly, the contraction coefficient: $\left(\frac{L}{\frac{1}{M}+c_{f} \varphi_{0}+L}\right)^{2}=\left(\frac{M \alpha^{2}}{2 \lambda+2 M \lambda c_{f} \varphi_{0}+\alpha^{2} M}\right)^{2}<1$, and independent of $q$.

\subsection{Convergence to discrete multirate formulation}

From the derivation above, we establish convergence of the sequences generated by the multirate fixed stress split algorithm and show that the converged quantities satisfy the weak formulation $(2.4)-(2.7)$. The proof uses the mathematical induction for the finer flow equations combined with the contraction estimates obtained above. 
Lemma 3.1 For every coarser mechanics time step, $t=t_{k}$, there exist a limit function $\boldsymbol{u}_{h}^{k}$ such that

$$
\boldsymbol{u}_{h}^{n, k} \rightarrow \boldsymbol{u}_{h}^{k} \quad \text { strongly in } H^{1}(\Omega)^{d}
$$

Proof. The contraction result in (3.21) implies that for a coarser time step $t=t_{k},\left\|\boldsymbol{\varepsilon}\left(\delta \boldsymbol{u}_{h}^{n+1, k}\right)\right\|$ converges geometrically to zero. This implies that $\boldsymbol{\varepsilon}\left(\boldsymbol{u}_{h}^{n+1, k}\right)$ is a Cauchy sequence converging geometrically to a unique limit in $L^{2}(\Omega)$. It follows immediately that $\boldsymbol{u}_{h}^{n+1, k}$ is a Cauchy sequence converging geometrically to a unique limit in $H^{1}(\Omega)^{d}$, being a Hilbert space.

Lemma 3.2 For every two consecutive coarser mechanics time steps, $t=t_{k}$, and $t=t_{k+q}$, and for every $1 \leq m \leq q$, there exist limit functions $p_{h}^{k+m}, \boldsymbol{z}_{h}^{k+m}$ such that

$$
p_{h}^{n, k+m} \rightarrow p_{h}^{k+m} \quad \text { in } L^{2}(\Omega), \quad \boldsymbol{z}_{h}^{n, k+m} \rightarrow \boldsymbol{z}_{h}^{k+m} \quad \text { in } H(d i v, \Omega)^{d},
$$

with strong convergence in the norms of the above spaces.

Proof. The contraction result in (3.21) implies that the quantities $\sum_{m=1}^{q} \| K^{-1 / 2}\left(\delta \boldsymbol{z}_{h}^{n+1, k+m}-\right.$ $\left.\delta \boldsymbol{z}_{h}^{n+1, k+m-1}\right) \|^{2}$, and $\sum_{m=1}^{q}\left\|\delta \sigma_{v}^{n+1, k+m}\right\|^{2}$ converge geometrically to zero. It follows that for $1 \leq m \leq q,\left\|K^{-1 / 2}\left(\delta \boldsymbol{z}_{h}^{n+1, k+m}-\delta \boldsymbol{z}_{h}^{n+1, k+m-1}\right)\right\|^{2}$, and $\left\|\delta \sigma_{v}^{n+1, k+m}\right\|^{2}$ converge geometrically to zero. Moreover, by (3.2), and Poincaré inequality, $\left\|K^{1 / 2} \nabla\left(\delta p_{h}^{n+1, k+m}-\delta p_{h}^{n+1, k+m-1}\right)\right\|^{2}$ and $\left\|\delta p_{h}^{n+1, k+m}-\delta p_{h}^{n+1, k+m-1}\right\|^{2}$ converge geometrically to zero, respectively. This implies that for every $1 \leq m \leq q$, the finer time step differences $\left(p_{h}^{n, k+m}-p_{h}^{n, k+m-1}\right),\left(\boldsymbol{z}_{h}^{n, k+m}-\boldsymbol{z}_{h}^{n, k+m-1}\right)$, and the volumetric mean stress defined by $\sigma_{v}^{n, k+m}$ are Cauchy sequences in $L^{2}(\Omega)$.

We will show strong convergence of the pressure sequence by induction. The proof of strong convergence of the flux sequence follows in the same way. Given an initial pressure value for $t=t_{0}: p_{h}^{n, 0}=p_{0}$, from the above discussion, $\left(p_{h}^{n, 1}-p_{0}\right)$ is a Cauchy sequence in $L^{2}(\Omega)$, and, in turn, $p_{h}^{n, 1}$ is a Cauchy sequence in the complete space $L^{2}(\Omega)$, and thus has a unique limit. This completes the base case for induction. For the inductive hypothesis, we assume that for any coarser mechanics time step $t=t_{k}$, and for any $1 \leq m \leq q, p_{h}^{n, k+m}$ is a Cauchy sequence converging to a unique limit in $L^{2}(\Omega): p_{h}^{n, k+m} \rightarrow p_{h}^{k+m}$ in $L^{2}(\Omega)$. We will show that $p_{h}^{n, k+m+1}$ is also a Cauchy sequence converging to a unique limit in $L^{2}(\Omega)$. However, this follows immediately, as $\left(p_{h}^{n, k+m+1}-p_{h}^{n, k+m}\right)$ is a Cauchy sequences in $L^{2}(\Omega)$, converging to a unique limit in $L^{2}(\Omega)$. This completes the inductive step. Therefore, we obtain that for all 
coarser mechanics time steps $t=t_{k}$, and for $1 \leq m \leq q, p_{h}^{n, k+m}, \boldsymbol{z}_{h}^{n, k+m}$ are Cauchy sequences converging geometrically to unique limits in $L^{2}(\Omega)$.

For the divergence of the flux, we note that (3.6) amounts to the following equality a.e. in $L^{2}(\Omega)$ :

$$
\nabla \cdot \delta \boldsymbol{z}_{h}^{n+1, k+m}=-\frac{\beta \mu_{f}}{\Delta t}\left(\delta p_{h}^{n+1, k+m}-\delta p_{h}^{n+1, k+m-1}\right)-\frac{\mu_{f} \chi}{\Delta t} \delta \sigma_{v}^{n, k+m} .
$$

The convergence of $\nabla \cdot \boldsymbol{z}_{h}^{n, k+m}$ in $L^{2}(\Omega)$ follows from the convergence of the difference $\left(p_{h}^{n, k+m}-\right.$ $p_{h}^{n, k+m}$ ) and $\sigma_{v}^{n, k+m}$ in $L^{2}(\Omega)$, established above. Thus, we have both $\nabla \cdot \boldsymbol{z}_{h}^{n, k+m}$ and $\boldsymbol{z}_{h}^{n, k+m}$ converging geometrically to unique limits in $L^{2}(\Omega)$, and hence $\boldsymbol{z}_{h}^{n+1, k}$ converges to a unique limit in $H(\text { div }, \Omega)^{d}$.

It remains to pass to the limit in (2.4)-(2.6). This is straightforward since the equations are linear and all operators involved are continuous in the spaces invoked in the statements of Lemmas 3.1 and 3.2. Moreover the convergences are strong. Therefore, we easily retrieve the discrete in time formulation.

The above discussions are summarized in the following main result:

Theorem 3.3 [Multirate (1)] For $L=\frac{\alpha^{2}}{2 \lambda}, \chi^{2}=L^{2}$, and $c_{0}=\frac{2 L}{q \chi^{2}}$, the multirate iterative scheme is a contraction given by

$$
\begin{aligned}
& 2 G c_{0}\left\|\boldsymbol{\varepsilon}\left(\delta \boldsymbol{u}_{h}^{n+1, k+q}\right)\right\|^{2}+\sum_{m=1}^{q}\left\|\delta \sigma_{v}^{n+1, k+m}\right\|^{2}+\frac{\Delta t}{\beta \mu_{f}}\left\|\boldsymbol{K}^{-1 / 2} \delta \boldsymbol{z}_{h}^{n+1, k+q}\right\|^{2} \\
& +\frac{\Delta t}{\beta \mu_{f}} \sum_{m=1}^{q}\left\|\boldsymbol{K}^{-1 / 2}\left(\delta \boldsymbol{z}_{h}^{n+1, k+m}-\delta \boldsymbol{z}_{h}^{n+1, k+m-1}\right)\right\|^{2} \leq\left(\frac{M \alpha^{2}}{2 \lambda+2 M \lambda c_{f} \varphi_{0}+\alpha^{2} M}\right)^{2} \sum_{m=1}^{q}\left\|\delta \sigma_{v}^{n, k+m}\right\|^{2} .
\end{aligned}
$$

Furthermore, the sequences defined by this scheme converge to the unique solution of the weak formulation (2.4)-(2.6).

Remark 3.4 We note that the contraction coefficient obtained in Theorem 3.3 exactly matches the contraction coefficient of the single rate optimized fixed stress split iterative method in the work of Mikelic and Wheeler [25]. Also, for the contraction results, we have assumed the compressibility term $\beta$ to be positive. In case of incompressibility limits ( $\beta$ approaching zero), the contraction coefficient tends to 1 . However, as the next remark shows we can still obtain contraction as long as the permeability remains positive. When $\lambda$ approaches infinity, there is no change in the porosity in the flow equation and the contraction coefficient tends to zero.

Remark 3.5 We can sharpen the contraction coefficient obtained in 3.3 as follows. By triangle's inequality, the quantity of contraction can be written as

$$
\left\|\delta \sigma_{v}^{n+1, m+k}\right\| \leq \frac{\alpha}{q \chi}\left\|\nabla \cdot \delta \boldsymbol{u}_{h}^{n+1, k+q}\right\|+\frac{L}{\chi}\left\|\delta p_{h}^{n+1, m+k}-\delta p_{h}^{n+1, m-1+k}\right\| .
$$


The volumetric strain term can be bounded by Korn's inequality. In addition, using Poincare inequality, the pressure term can be estimated by the flux. This leads to

$$
\begin{aligned}
& \sum_{m=1}^{q}\left\|\delta \sigma_{v}^{n+1, m+k}\right\|^{2} \leq C\left(\left\|\boldsymbol{\varepsilon}\left(\delta \boldsymbol{u}_{h}^{n+1, k+q}\right)\right\|^{2}+\sum_{m=1}^{q}\left\|\boldsymbol{K}^{-1 / 2}\left(\delta \boldsymbol{z}_{h}^{n+1, m+k}-\delta \boldsymbol{z}_{h}^{n+1, m-1+k}\right)\right\|^{2}\right) \\
& \quad \leq C\left(\left\|\boldsymbol{\varepsilon}\left(\delta \boldsymbol{u}_{h}^{n+1, k+q}\right)\right\|^{2}+\sum_{m=1}^{q}\left\|\boldsymbol{K}^{-1 / 2}\left(\delta \boldsymbol{z}_{h}^{n+1, m+k}-\delta \boldsymbol{z}_{h}^{n+1, m-1+k}\right)\right\|^{2}+\left\|\boldsymbol{K}^{-1 / 2} \delta \boldsymbol{z}_{h}^{n+1, k+q}\right\|^{2}\right) .
\end{aligned}
$$

for a constant $C>0$. Now, we define $\mathcal{I}_{q}^{n+1, k+q}$ as:

$$
\begin{aligned}
\mathcal{I}_{q}^{n+1, k+q}=2 G c_{0}\left\|\boldsymbol{\varepsilon}\left(\delta \boldsymbol{u}_{h}^{n+1, k+q}\right)\right\|^{2}+\frac{\Delta t}{\beta \mu_{f}} \sum_{m=1}^{q} & \left\|\boldsymbol{K}^{-1 / 2}\left(\delta \boldsymbol{z}_{h}^{n+1, m+k}-\delta \boldsymbol{z}_{h}^{n+1, m-1+k}\right)\right\|^{2} \\
& +\frac{\Delta t}{\beta \mu_{f}}\left\|\boldsymbol{K}^{-1 / 2} \delta \boldsymbol{z}_{h}^{n+1, k+q}\right\|^{2}
\end{aligned}
$$

Inequality (3.22) can be written as, for a generic constant $C>0$ :

$$
\sum_{m=1}^{q}\left\|\delta \sigma_{v}^{n+1, m+k}\right\|^{2} \leq C \mathcal{I}_{q}^{n+1, k+q}
$$

The improved contraction result, based on theorem 3.3, can be written as:

$$
\left(\frac{1}{C}+1\right) \sum_{m=1}^{q}\left\|\delta \sigma_{v}^{n+1, m+k}\right\|^{2} \leq\left(\frac{M \alpha^{2}}{2 \lambda+2 M \lambda c_{f} \varphi_{0}+\alpha^{2} M}\right)^{2} \sum_{m=1}^{q}\left\|\delta \sigma_{v}^{n, m+k}\right\|^{2}
$$

which lead to the improved contraction constant,

$$
\sum_{m=1}^{q}\left\|\delta \sigma_{v}^{n+1, m+k}\right\|^{2} \leq\left(\frac{C}{1+C}\right)\left(\frac{M \alpha^{2}}{2 \lambda+2 M \lambda c_{f} \varphi_{0}+\alpha^{2} M}\right)^{2} \sum_{m=1}^{q}\left\|\delta \sigma_{v}^{n+1, m+k}\right\|^{2}
$$

It is difficult to estimate $C$ in practice. However, we expect its value to increase with larger Lamé coefficients. The derivation above implies that the contraction coefficient obtained earlier is multiplied by a factor strictly less than one: $\frac{C}{C+1}<1$. We can conclude that the contraction estimate obtained in theorem 3.3 is sharper for $C \gg 1$ (as the damping factor $\frac{C}{C+1}<1$ approaches one in this case), and looser for smaller values of $C$. These computations highlight the impact of the extra positive terms on the left hand side of the contraction result obtained in Theorem 3.3. 


\section{Modified Multirate Scheme}

We introduce the modified multirate iterative coupling algorithm which results in Banach contraction on the volumetric mean total stress as defined by Mikelić and Wheeler [25] for the single rate fixed stress split iterative method. The algorithm involves a slight modification in the iterative coupling algorithm, in which we employ "successive corrections" in the flow problem (the corrections cancel out in the limit). We split the iterative coupling iteration into an even and odd iterations: in odd coupling iterations, we solve exactly the same mass balance equation solved in the single rate case, in contrast, for even coupling iterations, we add flux correction terms to the left and right hand sides of the mass balance equation. The idea is to correct for the flux, as we take finer time steps within one coarser mechanics time step, so that the summation of the finer flow equations over one coarser mechanics time step retrieves the weak formation of the single rate case, hence, deduce a contraction result similar to the one obtained by Mikelić and Wheeler [25] but for a fully discrete setting.

The modified multirate scheme gives a mechanism by which the multirate iterative coupling scheme is reduced to a corresponding single rate scheme, with a coarse time step for both flow and mechanics. Flux corrections terms correct the error introduced by not solving mechanics at every flow fine time step. This reduces the scheme into a single rate scheme, and as a result, all established theoretical results for the single rate case will be applicable here as well. Another aspect is the fact that in the modified multirate scheme, the quantity of contraction is independent of $q$ (the number of flow fine time steps within one coarse mechanics time step). This is due to the fact that the modified scheme contracts on the same volumetric mean total stress as defined in the single rate case [25]. This is not the case in the original multirate scheme, as volumetric strains are divided by $q$ in the quantity of contraction (3.14). This means that as the value of $q$ increases, volumetric strain contributions to the quantity of contraction gets reduced. This restriction is completely eliminated in the modified multirate iterative coupling scheme. 


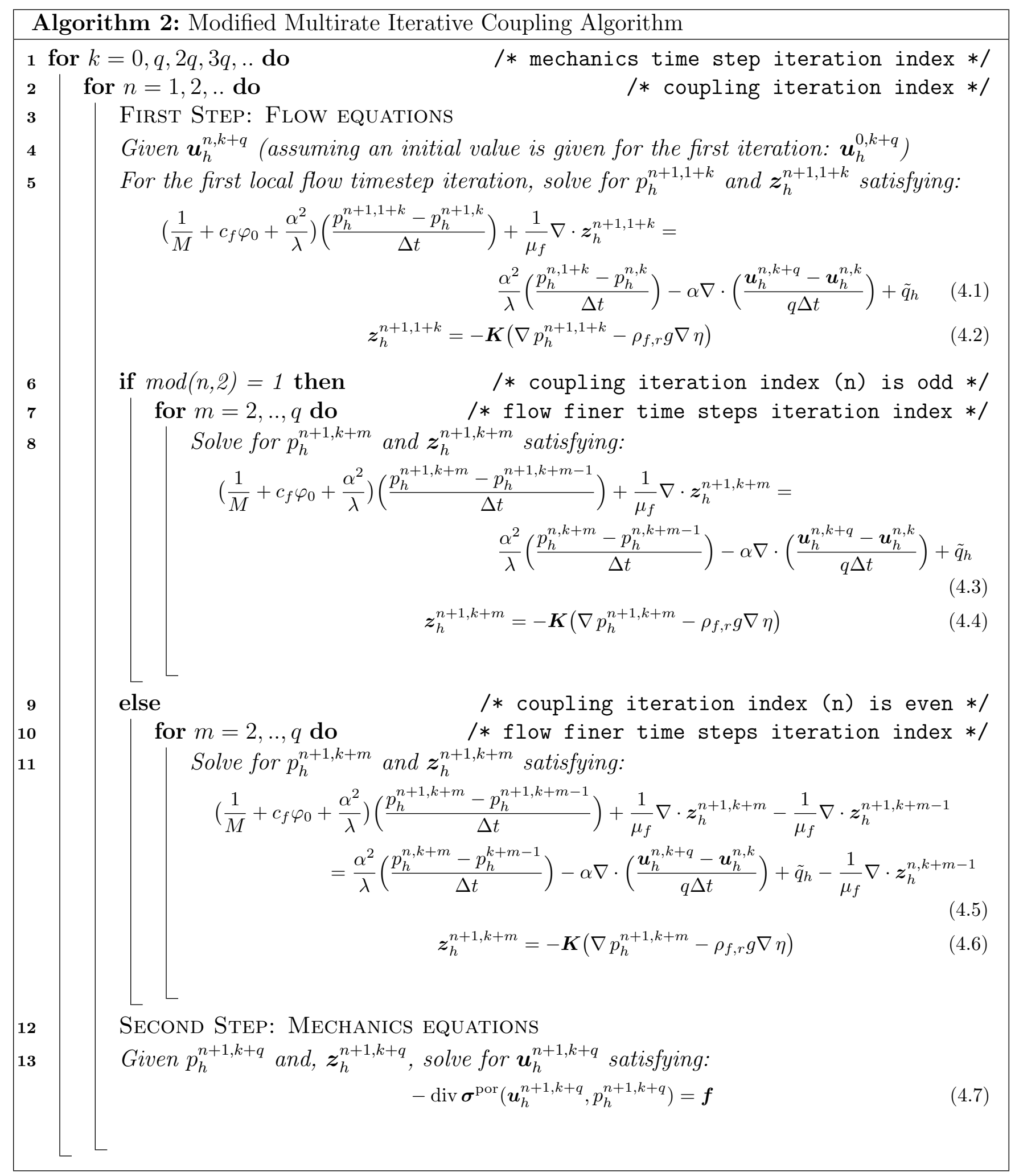


Remark 4.1 As indicated earlier, in contrast to the original multirate iterative coupling algorithm (Algorithm 1), in (Algorithm 2), we split the iterative coupling iterations into even and odd iterations. For the first finer flow time step, we solve exactly the same set of equations for both cases, as shown in line 5. For subsequent finer flow iterations, in the case of an even coupling iteration, we subtract flux correction terms, $\frac{1}{\mu_{f}} \nabla \cdot \boldsymbol{z}_{h}^{n+1, k+m-1}$, and $\frac{1}{\mu_{f}} \nabla \cdot \boldsymbol{z}_{h}^{n, k+m-1}$, from the left and right hand sides of the mass balance equation respectively, as shown in line 11. Upon convergence, $\boldsymbol{z}_{h}^{n+1, k+m-1}=\boldsymbol{z}_{h}^{n, k+m-1}$ and both terms cancel each other. In the case of an odd coupling iteration, we solve the same set of equations as in the single rate case, as shown in Line 8. With the newly introduced flux correction terms, a summation over finer time steps result in reducing the weak formulation of the multirate scheme to that of the single rate scheme. This allows us to obtain exactly the same contraction coefficient as the one obtained in the single rate case, Theorem 2.3. In addition, the modified scheme contracts on the volumetric mean total stress as defined in [25] for the single rate scheme.

\subsection{Proof of contraction of modified multirate scheme}

\section{- Step (1): Reduction to single rate weak formulation}

Extending the work of [25] to the fully discrete formulation, we define the volumetric mean stress, constituting the quantity to be contracted on, for $n \geq 1$, as:

$$
\begin{array}{ll}
\sigma_{v}^{n, k+m}=\sigma_{v}^{n, k}+\lambda \nabla \cdot \boldsymbol{u}_{h}^{n, k}-\alpha\left(p_{h}^{n, k+m}-p_{h}^{n, k}\right) & \text { for } 1 \leq m \leq q-1, \\
\sigma_{v}^{n, k+q}=\sigma_{v}^{n, k}+\lambda \nabla \cdot \boldsymbol{u}_{h}^{n, k+q}-\alpha\left(p_{h}^{n, k+q}-p_{h}^{n, k}\right) & \text { for } m=q .
\end{array}
$$

In terms of the difference between two coupling iterates, we have

$$
\begin{aligned}
& \delta \sigma_{v}^{n+1, k+m}=-\alpha \delta p_{h}^{n+1, k+m} \quad \text { for } 1 \leq m \leq q-1, \\
& \delta \sigma_{v}^{n+1, k+q}=\lambda \nabla \cdot \delta \boldsymbol{u}_{h}^{n+1, k+q}-\alpha \delta p_{h}^{n+1, k+q} \quad \text { for } m=q .
\end{aligned}
$$

In order to obtain the single rate weak formulation, we sum up local flow iterations across one coarser mechanics time step. As we solve different mass balance equations in even versus odd coupling iterations, we need to consider each case seperately:

- Coupling iteration index, $n$, is odd:

$$
\begin{gathered}
\left(\frac{1}{M}+c_{f} \varphi_{0}+\frac{\alpha^{2}}{\lambda}\right) \frac{1}{\Delta t} \sum_{m=1}^{q}\left(p_{h}^{n+1, k+m}-p_{h}^{n+1, k+m-1}\right)+\frac{1}{\mu_{f}} \nabla \cdot \sum_{m=1}^{q} \boldsymbol{z}_{h}^{n+1, k+m}= \\
\frac{\alpha^{2}}{\lambda \Delta t} \sum_{m=1}^{q}\left(p_{h}^{n, k+m}-p_{h}^{k+m-1}\right)-\frac{\alpha}{q \Delta t} \nabla \cdot \sum_{m=1}^{q}\left(\boldsymbol{u}_{h}^{n, k+q}-\boldsymbol{u}_{h}^{n, k}\right)+q \tilde{q}_{h} \\
\sum_{m=1}^{q} \boldsymbol{z}_{h}^{n+1, k+m}=-\boldsymbol{K} \nabla\left(\sum_{m=1}^{q} p_{h}^{n+1, k+m}\right)+\frac{1}{\mu_{f}} \boldsymbol{K} q \rho_{f, r} g \nabla \eta
\end{gathered}
$$




\section{- Coupling iteration index, $\mathrm{n}$, is even:}

Equation (4.13) remains unchanged. For (4.5), we have:

$$
\begin{aligned}
& \left(\frac{1}{M}+c_{f} \varphi_{0}+\frac{\alpha^{2}}{\lambda}\right) \frac{1}{\Delta t} \sum_{m=1}^{q}\left(p_{h}^{n+1, k+m}-p_{h}^{n+1, k+m-1}\right)+\frac{1}{\mu_{f}} \nabla \cdot \sum_{m=1}^{q} \boldsymbol{z}_{h}^{n+1, k+m} \\
& -\frac{1}{\mu_{f}} \nabla \cdot \sum_{w=1}^{q-1} \boldsymbol{z}_{h}^{n+1, w+k}=\frac{\alpha^{2}}{\lambda \Delta t} \sum_{m=1}^{q}\left(p_{h}^{n, k+m}-p_{h}^{k+m-1}\right)-\frac{\alpha}{q \Delta t} \nabla \cdot \sum_{m=1}^{q}\left(\boldsymbol{u}_{h}^{n, k+q}-\boldsymbol{u}_{h}^{n, k}\right) \\
& +q \tilde{q}_{h}-\frac{1}{\mu_{f}} \nabla \cdot \sum_{w=1}^{q-1} \boldsymbol{z}_{h}^{n, w+k}
\end{aligned}
$$

Assuming, without loss of generality, that " $n+1$ " represents an even coupling iteration, and " $n$ " represents an odd coupling iteration, subtracting (4.12) from (4.14) to form the difference between two consecutive coupling iterates, and taking advantage of (3.17), we derive

$$
\left(\frac{1}{M}+c_{f} \varphi_{0}+\frac{\alpha^{2}}{\lambda}\right) \frac{1}{\Delta t} \delta p_{h}^{n+1, k+q}+\frac{1}{\mu_{f}} \nabla \cdot \delta \boldsymbol{z}_{h}^{n+1, q+k}=-\frac{\alpha}{\lambda \Delta t} \delta \sigma_{v}^{n, k+q} .
$$

Equation (4.15) involves only coarser time step variables. Considering the modified multriate iterative coupling scheme as a single rate scheme, in which both the flow and mechanics problems share the coarser time step, the weak formulation in terms of the differences between coupling iterates reads

$$
\begin{aligned}
& \forall \theta_{h} \in Q_{h},\left(\frac{1}{\Delta t}\left(\frac{1}{M}+c_{f} \varphi_{0}+\frac{\alpha^{2}}{\lambda}\right) \delta p_{h}^{n+1, k+q}, \theta_{h}\right)+\frac{1}{\mu_{f}}\left(\nabla \cdot \delta \boldsymbol{z}_{h}^{n+1, k+q}, \theta_{h}\right)=\left(-\frac{\alpha}{\lambda \Delta t} \delta \sigma_{v}^{n, k+q}, \theta_{h}\right), \\
& \forall \boldsymbol{q}_{h} \in \boldsymbol{Z}_{h},\left(\boldsymbol{K}^{-1} \delta \boldsymbol{z}_{h}^{n+1, k+q}, \boldsymbol{q}_{h}\right)=\left(\delta p_{h}^{n+1, k+q}, \nabla \cdot \boldsymbol{q}_{h}\right), \\
& \forall \boldsymbol{v}_{h} \in V_{h}, 2 G\left(\boldsymbol{\varepsilon}\left(\delta \boldsymbol{u}_{h}^{n+1, k+q}\right), \boldsymbol{\varepsilon}\left(\boldsymbol{v}_{h}\right)\right)+\lambda\left(\nabla \cdot \delta \boldsymbol{u}_{h}^{n+1, k+q}, \nabla \cdot \boldsymbol{v}_{h}\right)-\alpha\left(\delta p_{h}^{n+1, k+q}, \nabla \cdot \boldsymbol{v}_{h}\right)=0 .
\end{aligned}
$$

\section{- Step (2): Flow equations}

Recall $\beta=\frac{1}{M \alpha^{2}}+\frac{c_{f}}{\alpha^{2}} \varphi_{0}+\frac{1}{\lambda}$; testing (4.16) with $\theta_{h}=\delta p_{h}^{n+1, k+q}$ and applying Young's inequality, we obtain

$$
\begin{aligned}
\frac{\beta}{\Delta t}\left\|\alpha \delta p_{h}^{n+1, k+q}\right\|^{2}+\frac{1}{\mu_{f}}\left(\nabla \cdot \delta \boldsymbol{z}_{h}^{n+1, k+q}, \delta p_{h}^{n+1, k+q}\right) & =\frac{1}{\Delta t}\left(-\alpha \epsilon \delta p_{h}^{n+1, k+q}, \frac{1}{\epsilon \lambda} \sigma_{v}^{n, k+q}\right) \\
& \leq \frac{1}{\Delta t}\left(\frac{\epsilon^{2}}{2}\left\|\alpha \delta p_{h}^{n+1, k+q}\right\|^{2}+\frac{1}{2 \epsilon^{2} \lambda^{2}}\left\|\delta \sigma_{v}^{n, k+q}\right\|^{2}\right) .
\end{aligned}
$$


The choice $\epsilon^{2}=\beta$ absorbs the pressure term on the right hand side by its corresponding term on the left hand side, leading to

$$
\beta\left\|\alpha \delta p_{h}^{n+1, k+q}\right\|^{2}+\frac{2 \Delta t}{\mu_{f}}\left(\nabla \cdot \delta \boldsymbol{z}_{h}^{n+1, k+q}, \delta p_{h}^{n+1, k+q}\right) \leq \frac{1}{\beta \lambda^{2}}\left\|\delta \sigma_{v}^{n, k+q}\right\|^{2} .
$$

Testing (4.17) with $\boldsymbol{q}_{h}=\delta \boldsymbol{z}_{h}^{n+1, k}$, we obtain

$$
\left(\boldsymbol{K}^{-1} \delta \boldsymbol{z}_{h}^{n+1, k}, \delta \boldsymbol{z}_{h}^{n+1, k}\right)=\left(\delta p_{h}^{n+1, k}, \nabla \cdot \delta \boldsymbol{z}_{h}^{n+1, k}\right) .
$$

Combining (4.20) with (4.19) leads to a sum of two positive squared norms on the right hand side of (4.19), in which the right hand side constitutes the quantity to be contracted on,

$$
\beta\left\|\alpha \delta p_{h}^{n+1, k+q}\right\|^{2}+\frac{2 \Delta t}{\mu_{f}}\left\|K^{-1 / 2} \delta \boldsymbol{z}_{h}^{n+1, k+q}\right\|^{2} \leq \frac{1}{\beta \lambda^{2}}\left\|\delta \sigma_{v}^{n, k+q}\right\|^{2} .
$$

\section{- Step (3): Elasticity equation}

Testing (4.18) with $\boldsymbol{v}_{h}=\delta \boldsymbol{u}_{h}^{n+1, k}$, we obtain

$$
2 G\left\|\boldsymbol{\varepsilon}\left(\delta \boldsymbol{u}_{h}^{n+1, k+q}\right)\right\|^{2}+\lambda\left\|\nabla \cdot \delta \boldsymbol{u}_{h}^{n+1, k+q}\right\|^{2}-\alpha\left(\delta p_{h}^{n+1, k+q}, \nabla \cdot \delta \boldsymbol{u}_{h}^{n+1, k+q}\right)=0 .
$$

Combining (4.21) with (4.22), we infer

$$
\begin{aligned}
& \left\{\left\|\alpha \delta p_{h}^{n+1, k+q}\right\|^{2}-2\left(\alpha \delta p_{h}^{n+1, k+q}, \lambda \nabla \cdot \delta \boldsymbol{u}_{h}^{n+1, k+q}\right)+\lambda^{2}\left\|\nabla \cdot \delta \boldsymbol{u}_{h}^{n+1, k+q}\right\|^{2}\right\} \\
& +4 G \lambda\left\|\boldsymbol{\varepsilon}\left(\delta \boldsymbol{u}_{h}^{n+1, k+q}\right)\right\|^{2}+\frac{2 \Delta t \lambda M \alpha^{2}}{\mu_{f}\left(M \alpha^{2}+\lambda\left(1+M c_{f} \varphi_{0}\right)\right)}\left\|K^{-1 / 2} \delta \boldsymbol{z}_{h}^{n+1, k+q}\right\|^{2} \\
& +\lambda^{2}\left\|\nabla \cdot \delta \boldsymbol{u}_{h}^{n+1, k+q}\right\|^{2} \leq\left(\frac{M \alpha^{2}}{\lambda+M \lambda c_{f} \varphi_{0}+M \alpha^{2}}\right)^{2}\left\|\delta \sigma_{v}^{n, k+q}\right\|^{2} .
\end{aligned}
$$

The first three terms form a square of the volumetric mean stress defined in (4.11), establishing the quantity of contraction for the next iterative coupling iteration, $n+1$, on the right hand side of (4.23), as

$$
\begin{aligned}
& \left\|\delta \sigma_{v}^{n+1, k+q}\right\|^{2}+\frac{2 \Delta t \lambda M \alpha^{2}}{\mu_{f}\left(M \alpha^{2}+\lambda\left(1+M c_{f} \varphi_{0}\right)\right)}\left\|K^{-1 / 2} \delta \boldsymbol{z}_{h}^{n+1, k+q}\right\|^{2}+4 G \lambda\left\|\boldsymbol{\varepsilon}\left(\delta \boldsymbol{u}_{h}^{n+1, k+q}\right)\right\|^{2} \\
& +\lambda^{2}\left\|\nabla \cdot \delta \boldsymbol{u}_{h}^{n+1, k+q}\right\|^{2} \leq\left(\frac{M \alpha^{2}}{\lambda+M \lambda c_{f} \varphi_{0}+M \alpha^{2}}\right)^{2}\left\|\delta \sigma_{v}^{n, k+q}\right\|^{2}
\end{aligned}
$$

with a contraction coefficient $\left(\frac{M \alpha^{2}}{\lambda+M \lambda c_{f} \varphi_{0}+M \alpha^{2}}\right)^{2}<1$. 


\subsection{Convergence to the discrete form}

In the next lemma, we establish convergence of the sequences generated by the modified multirate iterative coupling scheme for coarser mechanics time steps.

Lemma 4.2 For $k=0, q$, 2q, .., there exist limit functions $p_{h}^{k}, \boldsymbol{u}_{h}^{k}, \boldsymbol{z}_{h}^{k}$ such that

$$
p_{h}^{n, k} \rightarrow p_{h}^{k} \quad \text { in } L^{2}(\Omega), \quad \boldsymbol{u}_{h}^{n, k} \rightarrow \boldsymbol{u}_{h}^{k} \quad \text { in } H^{1}(\Omega)^{d}, \quad \boldsymbol{z}_{h}^{n, k} \rightarrow \boldsymbol{z}_{h}^{k} \quad \text { in } H(\operatorname{div}, \Omega)^{d},
$$

with strong convergence in the norms of the above spaces.

Proof. The contraction result in (4.24) implies that $\left\|\delta \sigma_{v}^{n+1, k}\right\|_{\Omega},\left\|\nabla \cdot \delta \boldsymbol{u}_{h}^{n+1, k}\right\|_{\Omega}$, and $\left\|K^{-1 / 2} \delta \boldsymbol{z}_{h}^{n+1, k}\right\|_{\Omega}$ converge geometrically to zero. This implies that $\sigma_{v}^{n+1, k}, \nabla \cdot \boldsymbol{u}_{h}^{n+1, k}$, and $\boldsymbol{z}_{h}^{n+1, k}$ are Cauchy sequences converging to unique limits in $L^{2}(\Omega)$. By $(4.11)$, we conclude that $p_{h}^{n, k}$ is a Cauchy sequence converging geometrically to a unique limit in $L^{2}(\Omega)$, being a Hilbert space.

For the displacements, (4.24) implies that $\boldsymbol{\varepsilon}\left(\delta \boldsymbol{u}_{h}^{n+1, k}\right)$ converges geometrically to 0 in $L^{2}(\Omega)$. It follows immediately that $\boldsymbol{u}_{h}^{n+1, k}$ converges geometrically to a unique limit in the Hilbert space $H^{1}(\Omega)^{d}$.

For the divergence of the flux, we note that (4.16) amounts to the following equality a.e. in $L^{2}(\Omega)$ :

$$
\nabla \cdot \delta \boldsymbol{z}_{h}^{n+1, k}=\frac{\mu_{f}}{\Delta t}\left(\frac{1}{M}+c_{f} \varphi_{0}+\frac{\alpha^{2}}{\lambda}\right) \delta p_{h}^{n+1, k}-\frac{\mu_{f} \alpha}{\lambda \Delta t} \delta \sigma_{v}^{n, k} .
$$

The convergence of $\nabla \cdot \boldsymbol{z}_{h}^{n+1, k}$ in $L^{2}(\Omega)$ follows from the convergences of $p_{h}^{n+1, k}$ and $\sigma_{v}^{n, k}$ in $L^{2}(\Omega)$. Therefore, we have both $\nabla \cdot \boldsymbol{z}_{h}^{n+1, k}$ and $\boldsymbol{z}_{h}^{n+1, k}$ converging geometrically in $L^{2}(\Omega)$, hence $\boldsymbol{z}_{h}^{n+1, k}$ converges in $H(d i v, \Omega)^{d}$. The existence of the limiting function in $H(\operatorname{div}, \Omega)^{d}$ follows from the completeness of the space.

It remains to pass to the limit in the weak formulation of (4.1)-(4.7). This is straightforward in view of the linearity of equations and strong convergences obtained.

Theorem 4.3 For coarser mechanics time steps, $k=0, q, 2 q, .$. , the modified multirate iterative scheme is a contraction given by

$$
\begin{aligned}
& \left\|\delta \sigma_{v}^{n+1, k}\right\|_{\Omega}^{2}+\frac{2 \Delta t \lambda M \alpha^{2}}{\mu_{f}\left(M \alpha^{2}+\lambda\left(1+M c_{f} \varphi_{0}\right)\right)}\left\|K^{-1 / 2} \delta \boldsymbol{z}_{h}^{n+1, k}\right\|_{\Omega}^{2}+4 G \lambda\left\|\varepsilon\left(\delta \boldsymbol{u}_{h}^{n+1, k}\right)\right\|_{\Omega}^{2}+\lambda^{2}\left\|\nabla \cdot \delta \boldsymbol{u}_{h}^{n+1, k}\right\|_{\Omega}^{2} \\
& \leq\left(\frac{M \alpha^{2}}{\lambda+M \lambda c_{f} \varphi_{0}+M \alpha^{2}}\right)^{2}\left\|\delta \sigma_{v}^{n, k}\right\|_{\Omega}^{2}
\end{aligned}
$$

Furthermore, the sequences defined by this scheme converge to the unique solution of the weak formulation of (4.1)-(4.7). 
Remark 4.4 All our obtained results remain valid when the multipoint flux mixed finite element method (MFMFE) [36,37] is used for flow discretization. For clarification, consider the modified multirate scheme. Using the MFMFE method for flow discretization, (4.24) translate to

$$
\begin{aligned}
& \left\|\delta \sigma_{v}^{n+1, k+q}\right\|^{2}+\frac{2 \Delta t \lambda M \alpha^{2}}{\mu_{f}\left(M \alpha^{2}+\lambda\left(1+M c_{f} \varphi_{0}\right)\right)}\left(K^{-1} \delta \boldsymbol{z}_{h}^{n+1, k+q}, \delta \boldsymbol{z}_{h}^{n+1, k+q}\right)_{Q}+4 G \lambda\left\|\boldsymbol{\varepsilon}\left(\delta \boldsymbol{u}_{h}^{n+1, k+q}\right)\right\|^{2} \\
& +\lambda^{2}\left\|\nabla \cdot \delta \boldsymbol{u}_{h}^{n+1, k+q}\right\|^{2} \leq\left(\frac{M \alpha^{2}}{\lambda+M \lambda c_{f} \varphi_{0}+M \alpha^{2}}\right)^{2}\left\|\delta \sigma_{v}^{n, k+q}\right\|^{2}
\end{aligned}
$$

where $\left(K^{-1} ., .\right)_{Q}$ is the quadrature rule defined in [36] for the MFMFE corresponding spaces. It was shown by Wheeler and Yotov in [36], and then extended to distorted quadrilaterals and hexahedra in [37], that for any $\boldsymbol{z}_{h} \in \boldsymbol{Z}_{h},\left(K^{-1} \boldsymbol{z}_{h}, \boldsymbol{z}_{h}\right)_{Q} \geq C\left\|\boldsymbol{z}_{h}\right\|^{2}$, for a constant $C>0$. This immediately leads to a similar contraction result. The same argument holds for previously derived results in the first multirate scheme described earlier. Similarly, our results can be extended to other multi point flux approximation control volume method, MFPA, when used for flow discretization [20, 21]. The coercivity of the bilinear form of the fluxes has been established in [21] for quadrilaterls, and in [20] for triangular elements using the broken Raviart-Thomas, and the lowest order Brezzi-Doulas-Marini spaces respectively.

\section{$5 \quad$ Numerical Results}

The first multirate iterative coupling algorithm (Algorithm 1) is implemented in the in-house reservoir simulator Integrated Parallel Accurate Reservoir Simulator (IPARS). As discussed above, the flow problem is solved using mixed method and the mechanics by conformal Galerkin (see $[32,34]$ for more details). In the mixed method for the flow, the flux unknowns are locally eliminated and a pressure stencil is obtained. The flux is then computed by post-processing. We consider three numerical experiments in this section: the Mandel's problem, the quarter wellbore problem, and a realistic field scale problem modeling the Frio field in Texas. The first numerical experiment, the Mandel's problem, is a standard benchmark problem and has an analytical solution and is used to validate the accuracy of our proposed scheme. It also highlights the expected trade-off between multirate-savings and maintaining an acceptable level of accuracy of the obtained numerical solution. The second problem, the quarter wellbore model, is used to study the sharpness of our derived theoretical contraction estimates against numerical observed values. The third problem applies our algorithm on Frio field, a field-scale problem, that includes a challenging reservoir geometry. 


\subsection{Convergence Stopping Critera}

The stopping criterion is based on the difference of two successive iterates of porosity. We denote

$$
\begin{aligned}
& \delta \varphi_{\text {flow }}^{n, k+q}=\left(\frac{1}{M}+\frac{\alpha^{2}}{\lambda}\right) \delta p_{h}^{n, k+q} \\
& \delta \varphi_{\text {mech }}^{n, k+q}=\alpha \nabla \cdot \delta \boldsymbol{u}_{h}^{n, k+q}+\frac{1}{M} \delta p_{h}^{n, k+q}
\end{aligned}
$$

The expression (5.2) is the standard definition of the fluid content of the medium [16]. The conver-

gence stopping criterion is defined as follows: $\left\|\frac{\varphi_{\text {mech }}^{n, k+\varphi_{\text {flow }}^{n, k+q}}}{\varphi_{\text {mech }}^{n, k+q}}\right\|_{L^{\infty}}=\left\|\frac{\alpha \nabla \cdot \boldsymbol{u}_{h}^{n, k+q}-\frac{\alpha^{2}}{\lambda} p_{h}^{n, k+q}}{\varphi_{m e c h}^{n, k+q}}\right\|_{L^{\infty}}<$ TOL. We set TOL $=5 . \mathrm{E}-7,1 . \mathrm{E}-4,1 . \mathrm{E}-10$, for the Mandel's problem, the quarter wellbore problem, and the Frio field model respectively. We note that specifying the coupling iteration convergence criterion, especially in the multirate case, is still a subject of research.

\subsection{Mandel's Problem}

The well-known Mandel's benchmark problem consists of a 2D saturated poroelastic slab of a rectangular shape with width $2 \mathrm{a}$ and height $2 \mathrm{~b}$. The sample is loaded by a constant compressive force, of intensity $2 \mathrm{~F}$, applied on the rigid impervious top and bottom plates $(y= \pm b)$. The slab can drain laterally, with stress-free lateral edges $x= \pm a$. The force is applied instantaneously at time $t=0$, and gravity is neglected in this setting [34]. The original configuration of the problem is shown in figure 5.1a. The problem highlights the necessity of incorporating a poroelastic model into existing reservoir simulators, as it captures the unexpected Mandel-Cryer effect, and is a standard problem for verifying the accuracy of poroelasticity algorithms [27].

The model considered here is the linearized quasi-static Biot model [24]. The initial and boundary conditions are given as:

$$
\begin{aligned}
& \left.p\right|_{t=0}=0,\left.\quad \boldsymbol{u}\right|_{t=0}=0,\left.\quad \boldsymbol{\sigma}(\boldsymbol{u})\right|_{t=0}=\left(\begin{array}{cc}
0 & 0 \\
0 & 0
\end{array}\right) \quad \text { in }(-a, a) \times(-b, b) \\
& p=0, \quad \boldsymbol{\sigma}(\boldsymbol{u}) \boldsymbol{e}^{1}=\boldsymbol{\sigma}_{11}=0, \quad \text { on } x= \pm a \\
& \boldsymbol{z}_{y}=0, \quad \boldsymbol{\sigma}_{12}=0, \quad \int_{-a}^{a} \boldsymbol{\sigma}_{22} d x=-2 F, \quad \boldsymbol{u}_{y}=\text { unknown constant, } \quad \text { on } y= \pm b
\end{aligned}
$$

Following a similar approach as in [24] and noting that $\boldsymbol{u}_{y}=\boldsymbol{u}_{y}(y, t)$, and $\boldsymbol{u}_{x}=\boldsymbol{u}_{x}(x, t)$, the impervious rigid plate condition (on $y= \pm b$ ), is replaced by:

$$
\boldsymbol{z}_{y}=0, \quad \boldsymbol{\sigma}_{12}=0, \quad \boldsymbol{u}_{y}=U_{y}( \pm b, t) \quad \text { on } y= \pm b
$$

where $U_{y}( \pm b, t)$ is the value obtained from the closed form solution of the Mandel's problem [24]. It should be noted that the problem is symmetric about the $\mathrm{x}$ and $\mathrm{y}$ axes. Therefore, only the 


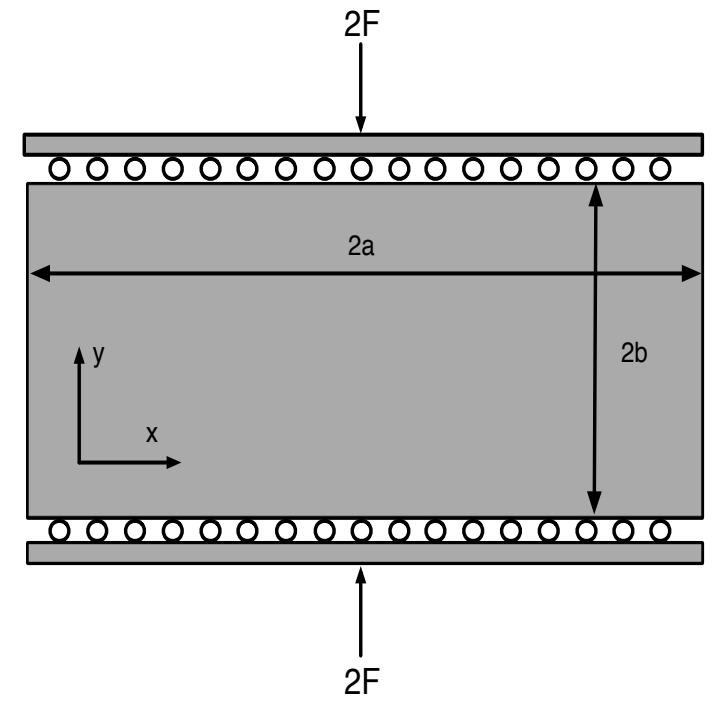

(a) Mandel's problem (original domain)

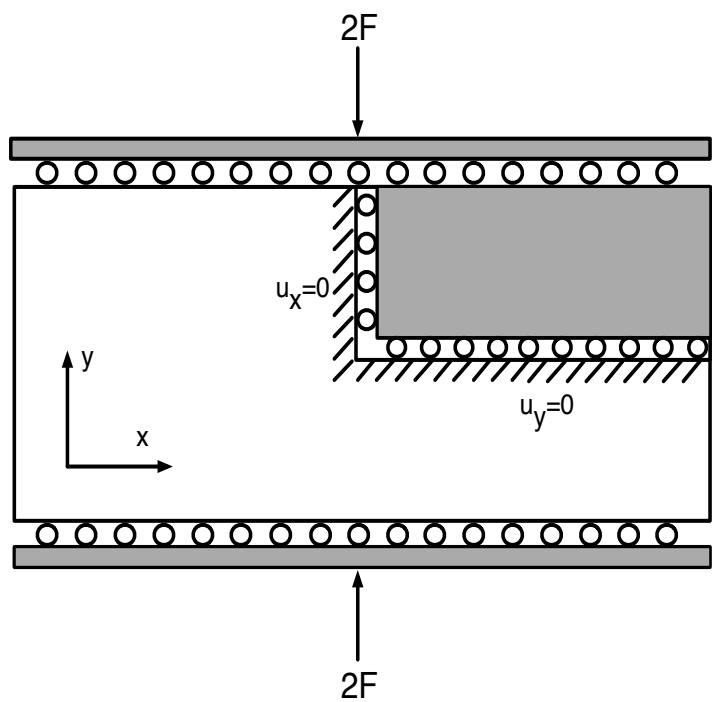

(b) Mandel's problem (computational domain)

Figure 5.1: Mandel's problem original and computational domains

upper-right quadrant $((0, a) \times(0, b))$ is going to be considered for numerical simulation, as shown in figure 5.1b. This restriction should not affect the initial conditions of the problem. However, the updated boundary conditions of the computational domain are given as follows:

$$
\begin{array}{ll}
p=0, & \boldsymbol{\sigma}(\boldsymbol{u}) \boldsymbol{e}^{1}=\boldsymbol{\sigma}_{11}=0, \quad \text { on } x=a \\
\boldsymbol{z}_{x}=0, & \boldsymbol{u}_{x}=0, \quad \boldsymbol{\sigma}_{12}=0, \quad \text { on } x=0 \\
\boldsymbol{z}_{y}=0, & \boldsymbol{\sigma}_{12}=0, \quad \boldsymbol{u}_{y}=U_{y}(b, t) \quad \text { on } y=b \\
\boldsymbol{z}_{y}=0, & \boldsymbol{u}_{y}=0, \quad \boldsymbol{\sigma}_{12}=0, \quad \text { on } y=0
\end{array}
$$

The original analytical solution of the problem, as given by Mandel [23], specifies a closed form solution of the pore-pressure only, for the isotropic case. Later, Abousleiman et al. [1] generalized the problem to include material transverse isotopy, with compressible solid constituents and pore fluid, and they presented detailed analytical solutions for pore pressure, displacements, and stresses [1]. We refer the reader to $[11,27]$ for the exact expressions of the parameters involved in this problem, including the Skempton pore pressure coefficient $B$, the fluid diffusivity coefficient $c$, the undrained Poisson ration $\nu_{u}$, and the analytical solutions including pore pressures $p$, displacements $\left(u_{x}, u_{y}\right)$, and stresses. For our numerical tests in this paper, the input parameters are shown in Table 1. 


\begin{tabular}{||cc||}
\hline Total Simulation time: & 50,000 seconds \\
\hline Finer $($ Unit) time step $(\Delta t):$ & 80 seconds \\
\hline Dimension in x $(a):$ & $328.084 \mathrm{ft}$ \\
\hline Dimension in y $(b):$ & $32.8084 \mathrm{ft}$ \\
\hline Number of grids: & 1600 grids $(40 \times 40)$ \\
\hline Grid spacing in x $(\Delta x):$ & $8.202 \mathrm{ft}$ \\
\hline Grid spacing in y $(\Delta y):$ & $0.8202 \mathrm{ft}$ \\
\hline Permeabilities: $\left(k_{x x}, k_{y y}\right)$ & $100,100 \mathrm{md}$ \\
\hline Initial porosity, $\left(\varphi_{0}\right)$ & 0.2 \\
\hline Fluid viscosity, $(\mu)$ & $1.0 \mathrm{cp}$ \\
\hline Fluid compressibility $\left(c_{f}\right)$ & $2.089 \mathrm{E}-6(1 / \mathrm{psi})$ \\
\hline Fluid density, $\left(\rho_{f, r}\right):$ & $62.4 l b_{m} / \mathrm{ft}^{3}$ \\
\hline Young's Modulus $(E)$ & $8.6152507 \mathrm{E}+5 \mathrm{psi}$ \\
\hline Possion Ratio, $(\nu)$ & 0.2 \\
\hline Undrained Possion Ratio, $\left(\nu_{u}\right)$ & 0.44 \\
\hline Biot's constant, $(\alpha)$ & 1.0 \\
\hline Biot Modulus, $(M)$ & $2.3931227 \mathrm{E}+6 \mathrm{psi}$ \\
\hline Skempton coefficient, $(B)$ & 0.8333 \\
\hline Diffusivity coefficient, $(c)$ & $5.0052 \mathrm{ft} t^{2} / s$ \\
\hline Introduced Fixed Stress Parameter $(L)$ & $\frac{\alpha^{2}}{2 \lambda}$ \\
\hline
\end{tabular}

Table 1: Input Parameters for the Mandel's Problem

\subsubsection{Results}

Figures 5.3a, 5.3b, 5.3c, and 5.3d show numerical versus analytical results for the pressure variable at times $t=640,1280,5120,10240$, and 20480 seconds, for $q=1,2,4$, and 8 respectively. Results are most accurate for the single rate case $q=1$, and accuracy degrades slightly as the value of $q$ increases, which is expected. A similar behaviour, although at a much smaller scale, for x-displacements as shown in figures $5.4 \mathrm{a}, 5.4 \mathrm{~b}, 5.4 \mathrm{c}$, and $5.4 \mathrm{~d}$, for $q=1,2,4$, and 8 respectively. CPU run times for the whole simulation run (50,000 seconds) are shown in figure 5.2a. For $q=2$, we save $20.86 \%$ in CPU runtime. For $q=4$, and 8, we save $42.51 \%$ and $60.09 \%$ in CPU runtime respectively. Runtime savings can be attributed to the huge reductions in the total number of mechanics linear iterations for multirate cases. For $q=2,4$, and 8, the total number of mechanics linear iterations for the whole simulation run went down by $38.15 \%, 65.25 \%$, and $82.43 \%$ respectively. Tables 2 and 3 show the accuracy of the obtained solution against the analytical solution at two time steps during the simulation run $(t=640$, and 20480) seconds respectively. We see that for both time steps, the accuracy of pressures degrades only slightly. Similarly, for displacements, the accuracy is only slightly affected. The numerical tests demonstrate that the multirate scheme maintains the accuracy of the solution whereas providing significant computational advantages. 


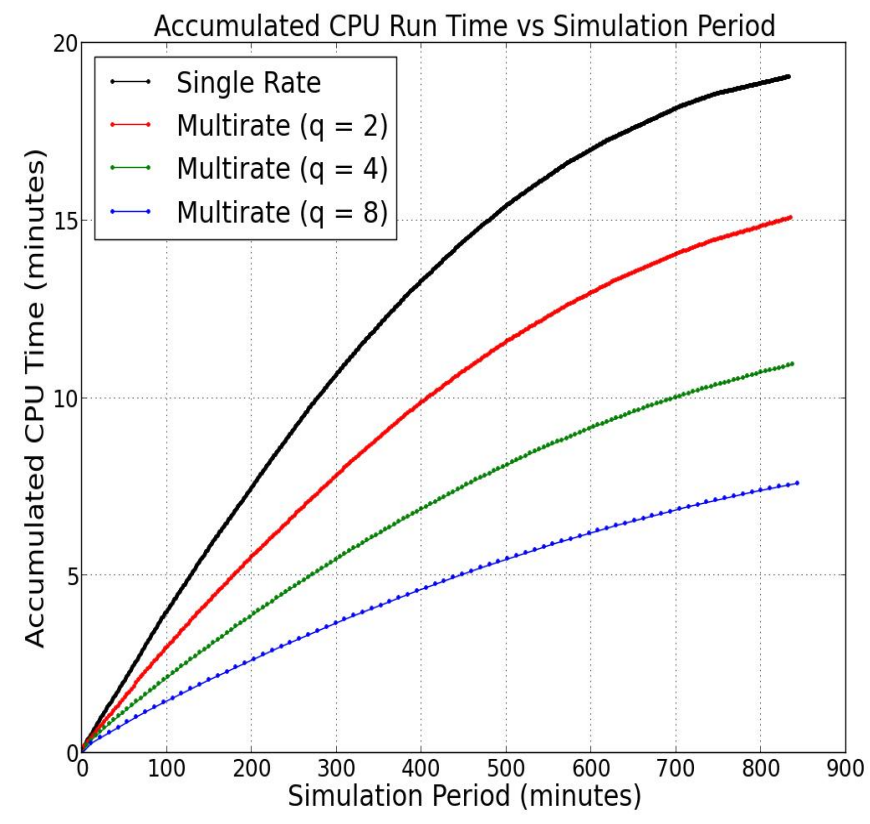

(a) CPU Run Time vs Simulation Days

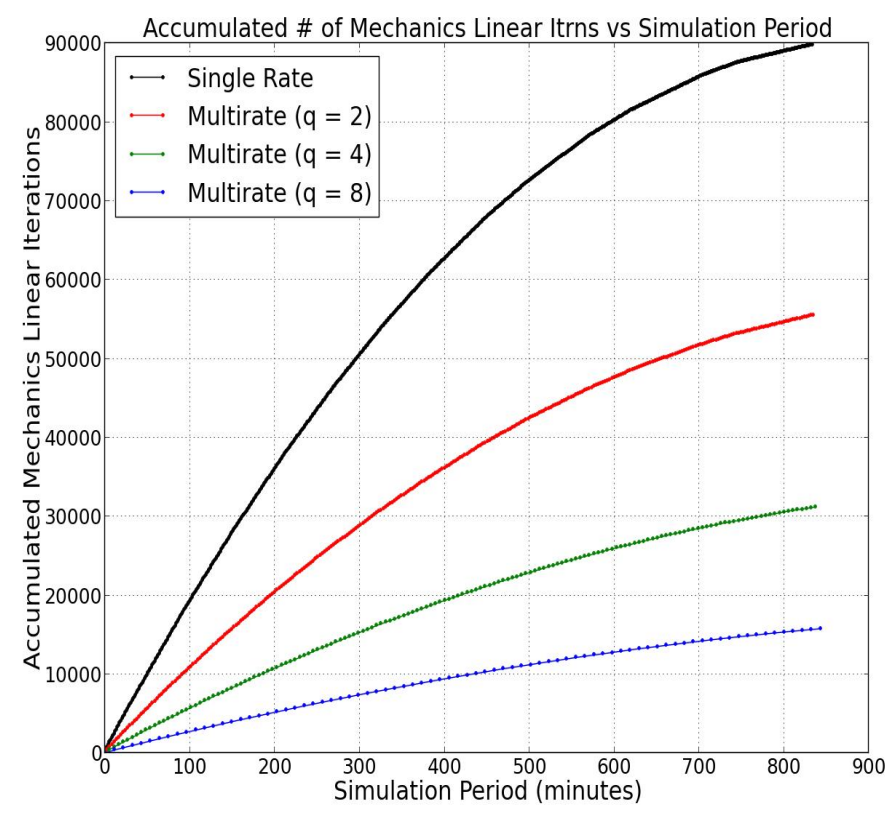

(b) Total Number of Mechanics Linear Iterations vs Simulation Days

Figure 5.2: Mandel's Problem Multirate Savings

\begin{tabular}{||c|c|c|c|c||}
\cline { 2 - 5 } \multicolumn{1}{c||}{} & $q=1$ & $q=2$ & $q=4$ & $q=8$ \\
\hline \hline$\left\|p_{h}-p(t)\right\|_{\ell_{2}}^{2}$ & $1.0863 \mathrm{e}-03$ & $1.6490 \mathrm{e}-03$ & $3.3955 \mathrm{e}-03$ & $7.9480 \mathrm{e}-03$ \\
\hline$\left\|u_{x_{h}}-u_{x}(t)\right\|_{\ell_{2}}^{2}$ & $9.1711 \mathrm{e}-12$ & $3.4294 \mathrm{e}-11$ & $3.4218 \mathrm{e}-10$ & $1.6005 \mathrm{e}-09$ \\
\hline Reduction in CPU Runtime & - & $16.35 \%$ & $27.90 \%$ & $45.78 \%$ \\
\hline Reduction in Mechanics linear iterations & - & $42.41 \%$ & $67.61 \%$ & $83.46 \%$ \\
\hline \hline
\end{tabular}

Table 2: Accuracy versus efficiency for different values of $q$ (the number of flow finer time steps within one coarser mechanics time step) at time $t=640$ seconds. Discrete $\ell_{2}$ norms are computed over the top boundary of the domain $(y=b)$, as $\mathrm{x}$-displacements depend only on $\mathrm{x}$-co-ordiantes.

$\mathrm{CPU}$ time savings and reductions in the number of mechanics linear iterations are computed against the single rate case $(q=1)$. 


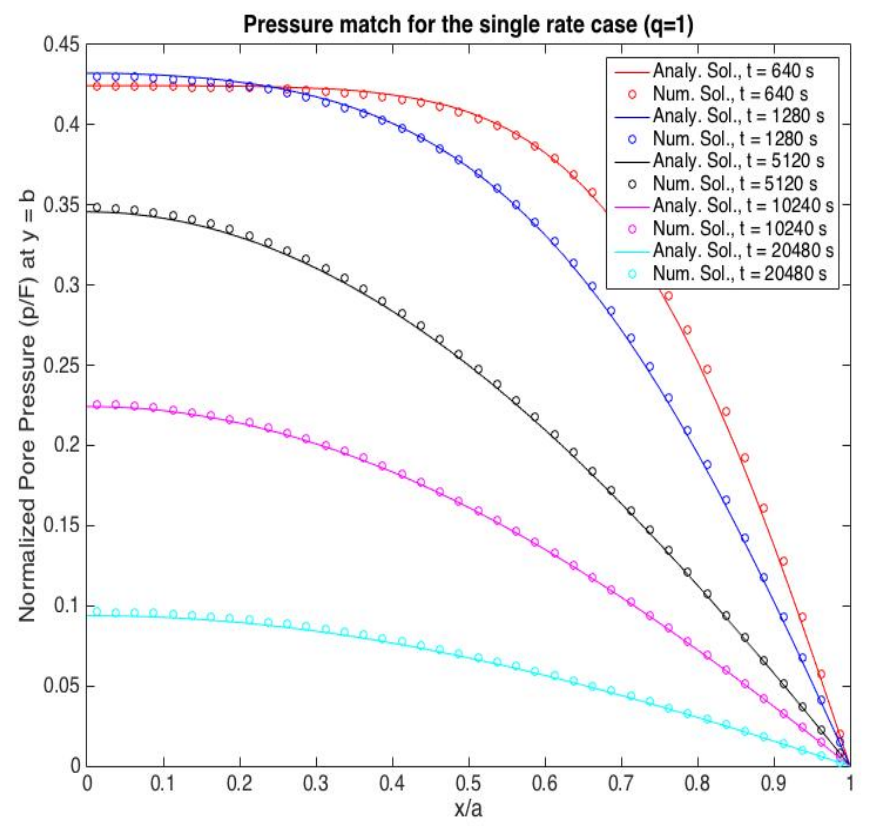

(a) Analytical vs Numerical Results $(q=1)$

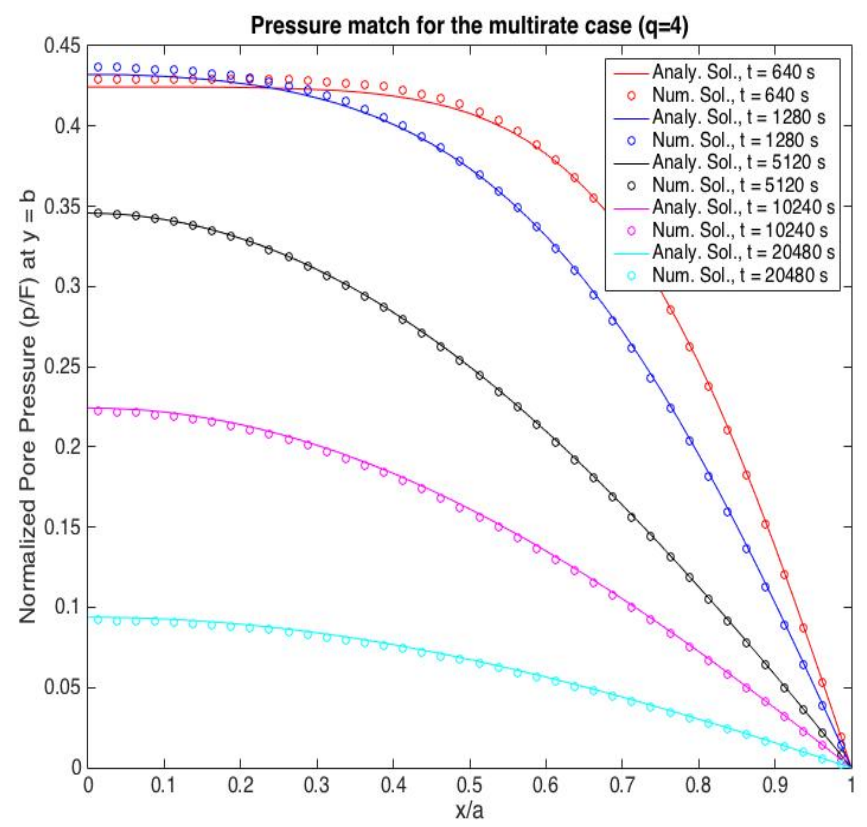

(c) Analytical vs Numerical Results $(q=4)$

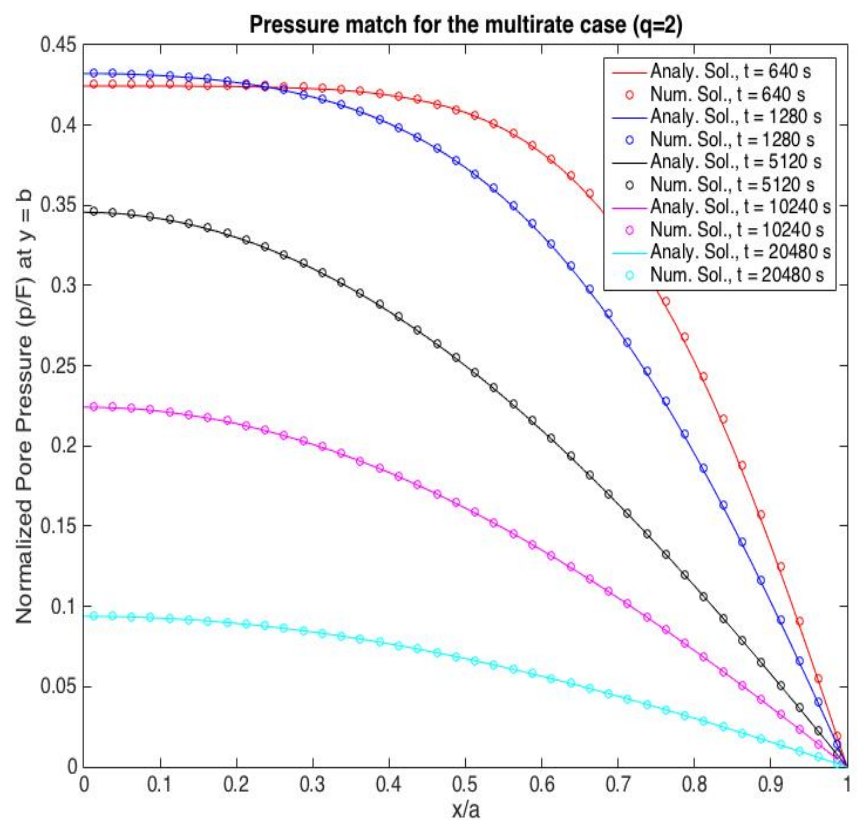

(b) Analytical vs Numerical Results ( $q=2)$

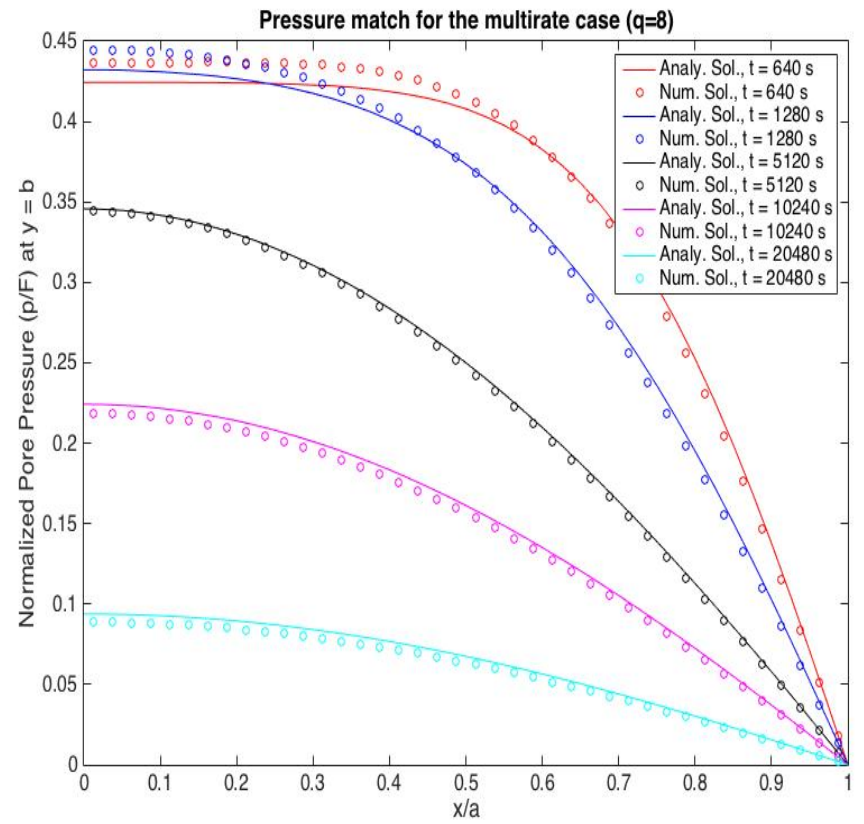

(d) Analytical vs Numerical Results ( $q=8)$

Figure 5.3: Accuracy of our Multirate Scheme on Mandel's Problem Pressure Solution 


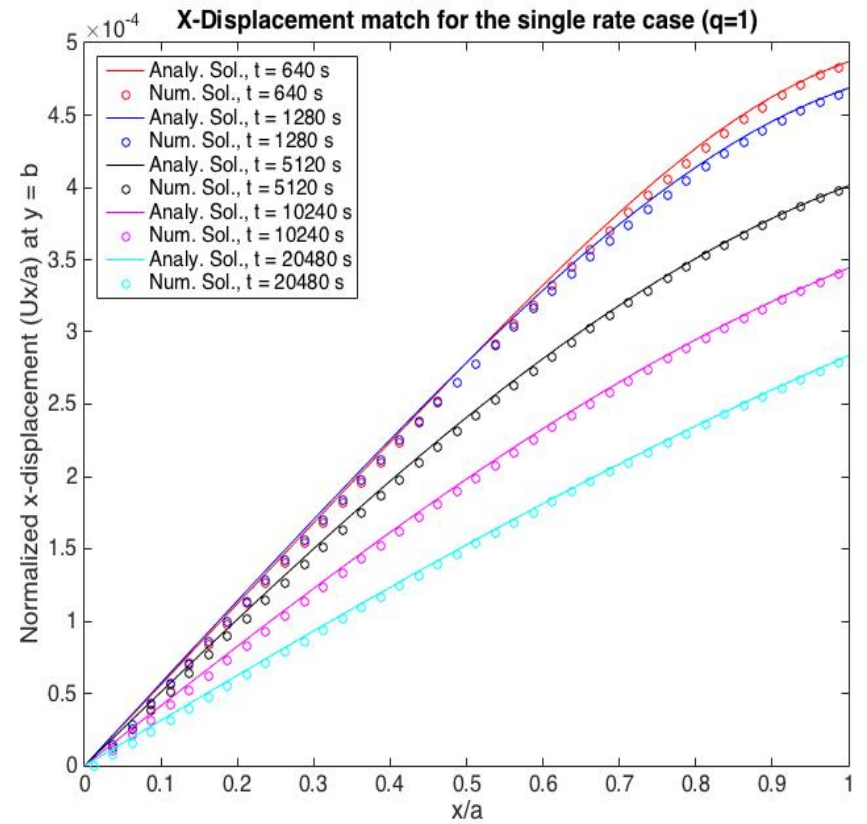

(a) Analytical vs Numerical Results ( $q=1)$

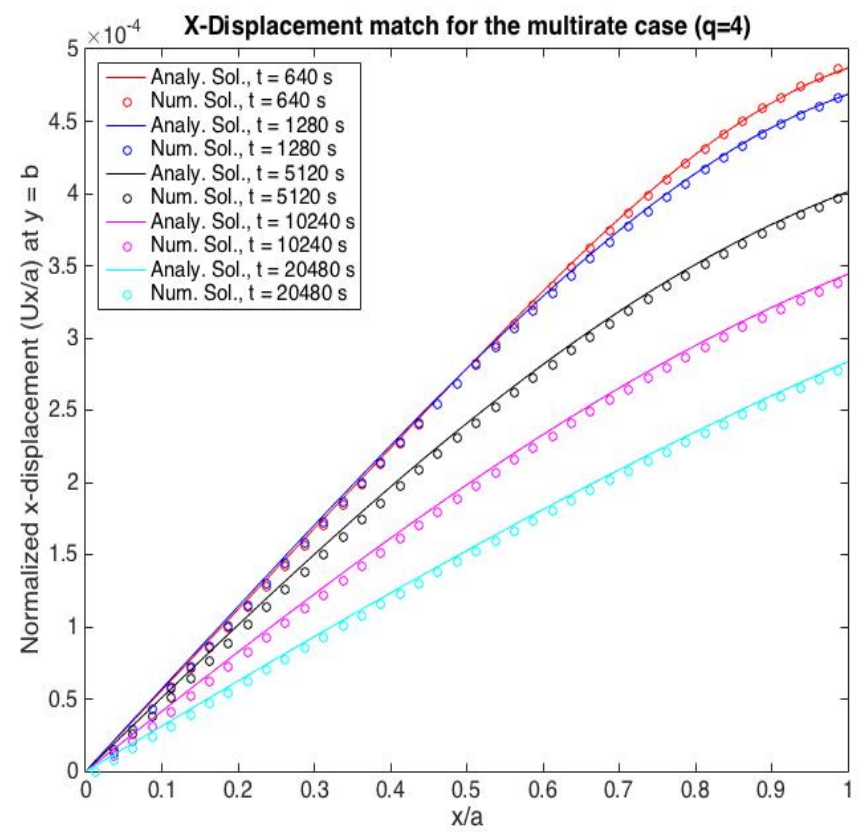

(c) Analytical vs Numerical Results ( $q=4)$

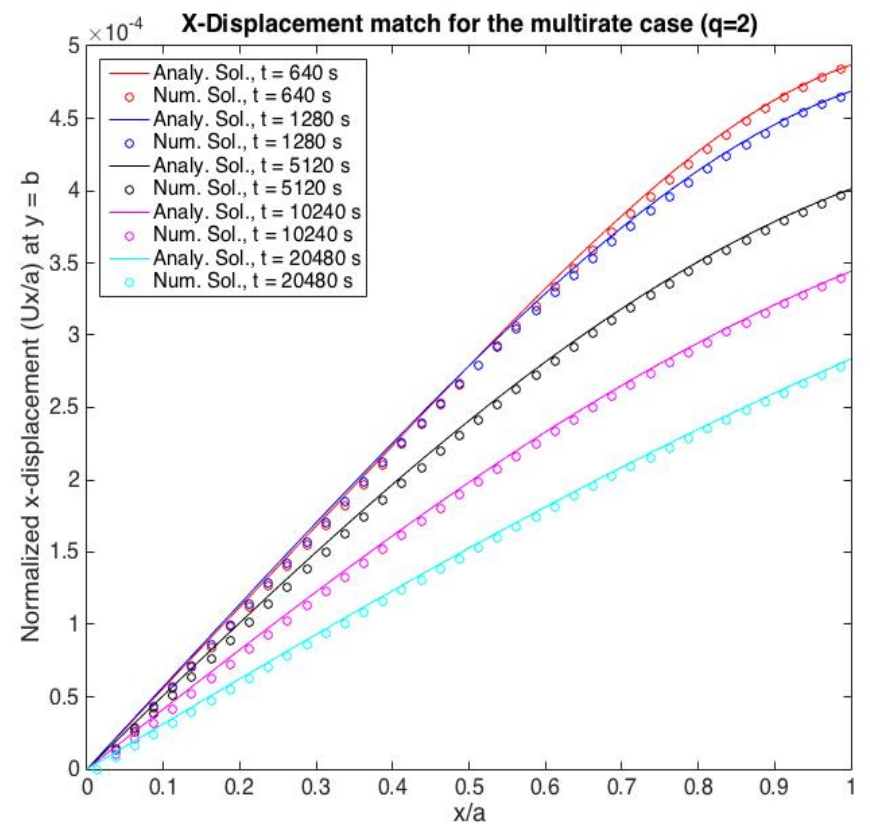

(b) Analytical vs Numerical Results ( $q=2$ )

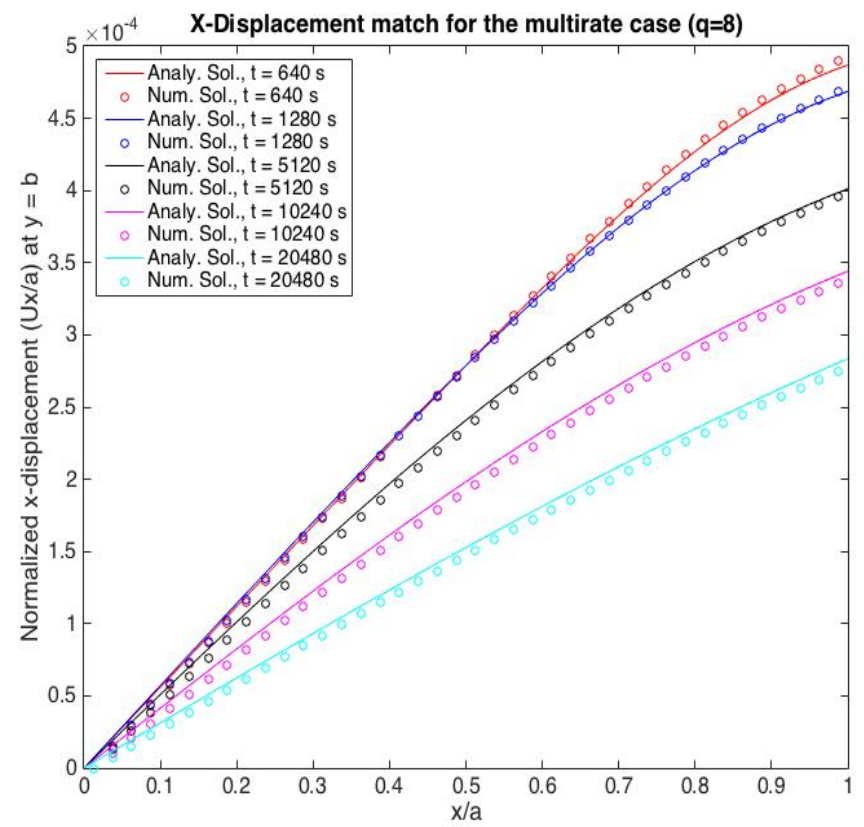

(d) Analytical vs Numerical Results ( $q=8)$

Figure 5.4: Accuracy of our Multirate Scheme on Mandel's Problem Displacement Solution 


\begin{tabular}{||c||c|c|c|c||}
\cline { 2 - 5 } \multicolumn{1}{c|}{} & $q=1$ & $q=2$ & $q=4$ & $q=8$ \\
\hline \hline$\left\|p_{h}-p(t)\right\|_{\ell_{2}}^{2}$ & $5.9838 \mathrm{e}-05$ & $6.1103 \mathrm{e}-05$ & $2.3748 \mathrm{e}-04$ & $9.2265 \mathrm{e}-04$ \\
\hline$\left\|u_{x_{h}}-u_{x}(t)\right\|_{\ell_{2}}^{2}$ & $3.1261 \mathrm{e}-11$ & $5.1934 \mathrm{e}-13$ & $1.6641 \mathrm{e}-11$ & $1.4160 \mathrm{e}-10$ \\
\hline Reduction in CPU Runtime & - & $26.27 \%$ & $48.57 \%$ & $65.51 \%$ \\
\hline Reduction in Mechanics linear iterations & - & $42.71 \%$ & $69.50 \%$ & $85.20 \%$ \\
\hline \hline
\end{tabular}

Table 3: Accuracy versus efficiency for different values of $q$ (the number of flow finer time steps within one coarser mechanics time step) at time $t=20480$ seconds.

\begin{tabular}{||cc||}
\hline Total Simulation time: & 1.024 days \\
\hline Finer (Unit) time step: & 0.0001 days \\
\hline Number of grids: & 4200 grids $(7 \times 20 \times 30)$ \\
\hline Permeabilities: $k_{x x}, k_{y y}, k_{z z}$ & $5,20,20 \mathrm{md}$ \\
\hline Initial porosity, $\varphi_{0}$ & 0.2 \\
\hline Fluid viscosity, $\mu_{f}$ & $1.0 \mathrm{cp}$ \\
\hline Initial pressure, $p_{0}$ & $500.0 \mathrm{psi}$ \\
\hline Fluid compressibility $c_{f}:$ & $1 . \mathrm{E}-6(1 / \mathrm{psi})$ \\
\hline Rock compressibility: & $1 . \mathrm{E}-6(1 / \mathrm{psi})$ \\
\hline Rock density: & $165.43 l b_{m} / \mathrm{ft}^{3}$ \\
\hline Initial fluid density, $\rho_{w}:$ & $62.34 l b_{m} / \mathrm{ft}^{3}$ \\
\hline Young's Modulus $(\mathrm{E})$ & $1.1 \mathrm{E} 7 \mathrm{psi}$ \\
\hline Possion Ratio, $\nu$ & 0.4 \\
\hline Biot's constant, $\alpha$ & 0.75 \\
\hline Biot Modulus, $M$ & $0.5 \mathrm{E} 14$ \\
\hline$L$ (introduced fixed stress parameter) & $\frac{\alpha^{2}}{2 \lambda}$ \\
\hline
\end{tabular}

Table 4: Input Parameters for the Quarter Wellbore Model

\subsection{Quarter Wellbore Model}

The second problem we consider is a quarter 3D wellbore model. The model domain is a $25.0 \mathrm{ft}$ $\times 25.0 \mathrm{ft} \times 25.0 \mathrm{ft}$ cube with a quarter of a cylindrical wellbore centered along one of its edges. The mesh contains 4200 grid elements, with 30 elements in the radial direction, 20 elements in the hoop direction, and 7 elements in the vertical direction. Finer grids are used near the wellbore, and they coarsen as they distance apart from the wellbore. A constant wellbore pressure of 300 psi is enforced on the wellbore surface. No flow boundary conditions are enforced on the rest of the boundary faces. For the mechanics model, we apply a zero displacement boundary condition on top of the cube. For the remaining boundaries, we apply zero normal and zero shear traction boundary conditions. Gravity is neglected in this model. Detailed specifications of the input parameters can be found in Table 4. 


\begin{tabular}{||l||c|c|c|c||}
\cline { 3 - 5 } & $q=1$ & $q=2$ & $q=4$ & $q=8$ \\
\hline \hline$\sum_{m=1}^{q}\left\|\delta \sigma_{v}^{3, m}\right\|_{/ \sum_{m=1}^{q}\left\|\delta \sigma_{v}^{2, m}\right\|^{2}}^{2}$ & 0.0009485 & 0.0007602 & 0.0004718 & 0.0001791 \\
\hline
\end{tabular}

Table 5: Numerical Contraction Estimates: Contraction estimates observed numerically are shown for different values of $q$ (the number of flow finer time steps within one coarser mechanics time step). These are obtained by taking the ratio of the norms of $\sigma_{v}$ computed at the last two iterative coupling iterations during the first coarse time step: $\Delta t, 2 \Delta t, 4 \Delta t$, and $8 \Delta t$ for $q=1,2$, 4 , and 8 respectively. The first coarse time step involves four iterative coupling iterations for all the four cases.

\subsubsection{Results \& Discussion}

Figure 5.5a shows the accumulated CPU run time for the single rate case $(q=1)$, and for multirate cases: $q=2,4$, and 8 . The case $q=2$ results in $14.28 \%$ reduction in CPU run time compared to the single rate. $q=4$, and $q=8$ result in $20.97 \%$ and $25.09 \%$ reductions in CPU run times respectively. Figure 5.5c explains the reduction in CPU run time observed in the multirate case. By just solving for two flow finer time steps within one coarser mechanics time step $(q=2)$, the total number of mechanics linear iterations was reduced by $45.21 \%$ with reference to the single rate case. Multirate couplings $(q=4$, and $q=8)$ result in $70.46 \%$ and $84.36 \%$ reductions in the number of mechanics linear iterations respectively, which in turn, reduce the CPU run time as well. For this problem, the total number of flow iterations for both the single rate and multirate coupling algorithms are found to be the same. In addition, all four cases perform the same number of flow/mechanics coupling iterations for each coarse mechanics time step, reducing the number of accumulated mechanics linear iterations for multirate schemes, without affecting the total number of flow linear iterations. This results in multirate coupling schemes to outperform the single rate scheme.

We also compare the value of our theoretically driven contraction coefficient against numerically observed contraction coefficient values. Theorem 3.3 gives an expression of the contraction coefficient $\left(\frac{M \alpha^{2}}{2 \lambda+2 M \lambda c_{f} \varphi_{0}+M \alpha^{2}}\right)^{2}$ for the multirate algorithm considered in this case $\left(L=\frac{\alpha^{2}}{2 \lambda}\right)$, leading to linear convergence of the multirate scheme. For this test case, we have $\left(\frac{M \alpha^{2}}{2 \lambda+2 M \lambda c_{f} \varphi_{0}+M \alpha^{2}}\right)^{2}=0.006747$. Table 5 lists the values of contraction coefficients obtained numerically for $q=1,2,4$, and 8 . We consider the iterative coupling iteration for the first coarse mechanics time step, which takes four coupling iterations to converge, according to the stopping criteria described earlier. We compute the values of the volumetric mean stress defined in (3.14) for the last two coupling iterations. Ratios of those computed values give estimates of contraction coefficients, obtained numerically, as shown in Table 5. We notice that contraction coefficients computed numerically are smaller than 


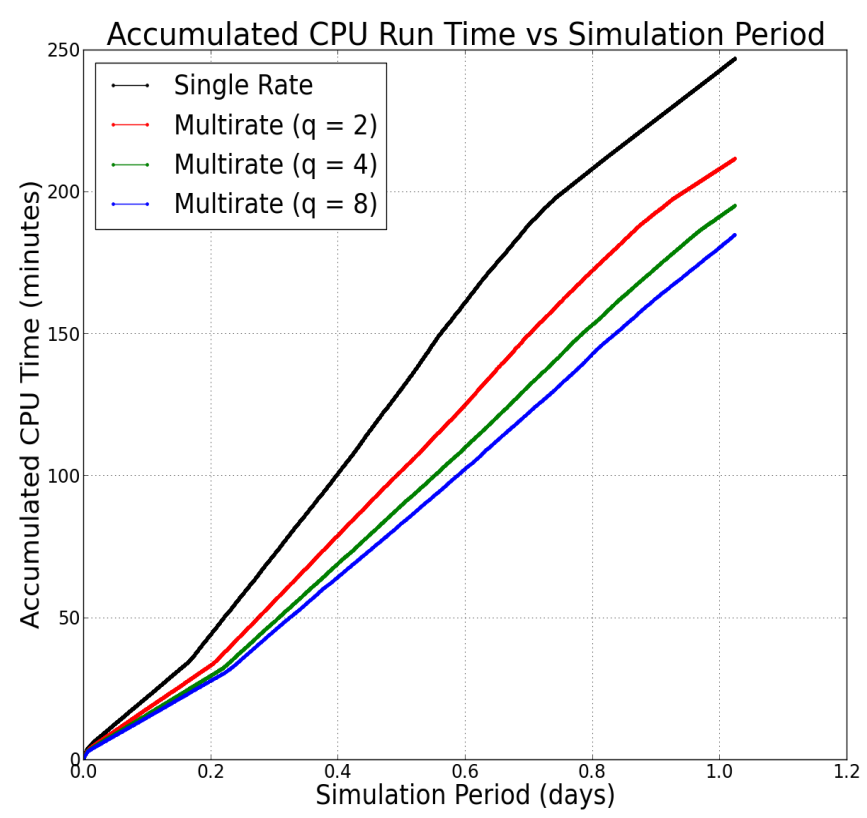

(a) CPU Run Time vs Simulation Days

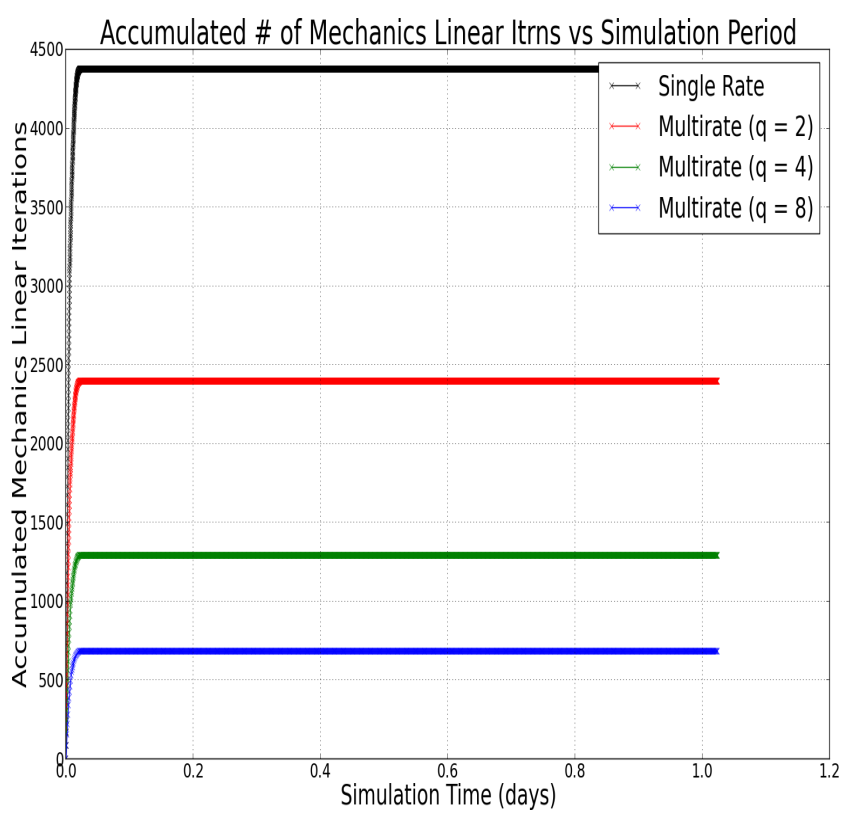

(b) Total Number of Mechanics Linear Iterations vs Simulation Days

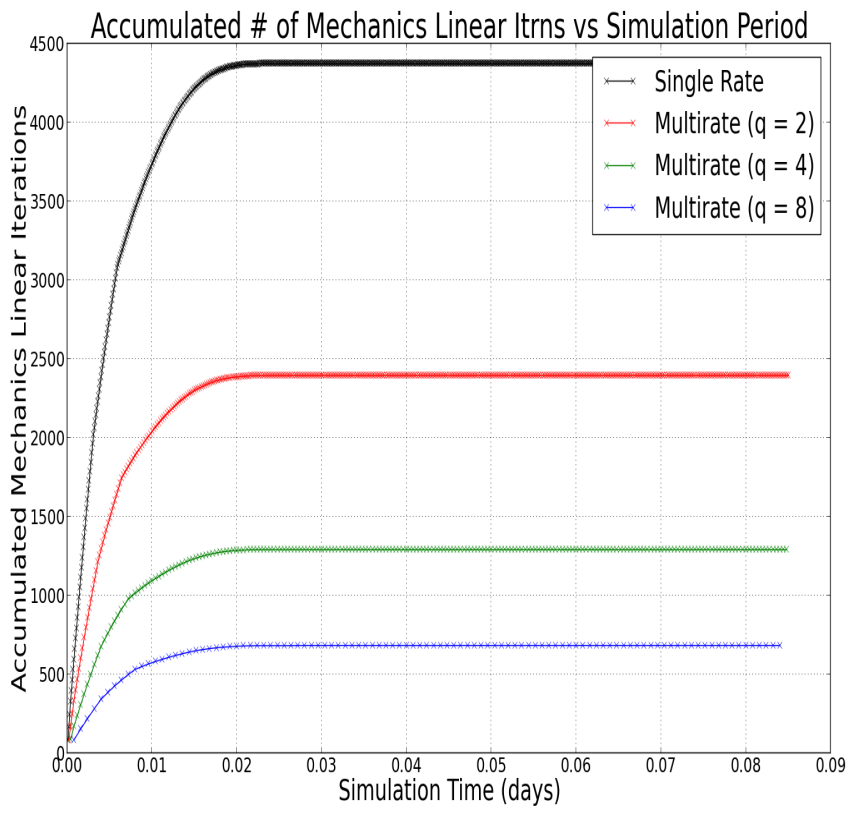

(c) Total Number of Mechanics Linear Iterations vs Simulation

Days (for initial time steps)

Figure 5.5: Quarter Wellbore Model: The black curve corresponds to the single rate case $(q=1)$. The red curve corresponds to the multirate case $(q=2)$. The green and blue curves correspond to multirate cases $(q=4$, and 8$)$ respectively. 
the predicted theoretical estimate. This is expected since the extra terms on the left hand side of the contraction result listed in theorem 3.3 are not included when computing numerical estimates. We notice that as the number of flow finer time steps solved within one coarser mechanics time step increases, the values of the computed numerical contraction coefficient estimates decrease.

\subsection{Frio Field Model}

The third test problem is a realistic field-scale reservoir model, located near Dayton, Texas, at South Liberty oil field on the Gulf Coast. The field contains several geometrically challenging thin curved faults, and is curved in the depth direction [17]. In this work, we try to consider the challenging geometry of the field along with its permeability distribution. Gravity effects are included in the model, and other input parameters are shown in Table 6.

\subsubsection{Results}

Pressure profiles and displacement vector fields for the single rate scheme, and multirate schemes $(q=4$ and 8$)$ after 480 simulation days are shown in figures 5.6a and 5.6b respectively. For all three cases $(q=1,4$, and 8), results are almost identical. Accumulated CPU runtimes for the three cases are shown in figure 5.7a. Multirate schemes $(q=4$ and 8$)$ result in $20.43 \%$, and $34.91 \%$ reductions in CPU run times respectively. Reductions in CPU run times come as a direct consequence of the huge reductions in the accumulative number of mechanics linear iterations for the whole simulation run. For $q=4$, the total number of mechanics linear iterations is reduced by $58.88 \%$, and for $q=8$, mechanics linear iterations are reduced by 79.44\%. The overhead introduced by the multirate coupling scheme over the the single rate scheme is illustrated in figures 5.7c and 5.7d. As shown in figure $5.7 \mathrm{c}$, multirate schemes $(\mathrm{q}=4)$, and $(\mathrm{q}=8)$ result in $92.26 \%$, and $93.40 \%$ increase in the total number of flow linear iterations for the whole simulation run. This overhead is attributed to the observed increase in the number of flow-mechanics coupling iterations for multirate schemes over the single rate scheme, as shown in figure $5.7 \mathrm{~d}$. It should be noted that, with respect to running times, the decrease in the number of mechanics linear iterations outperform the overhead introduced by the increase in the total number of flow linear iterations. It is this particular feature that allows the multirate scheme to outperform the single rate scheme with respect to CPU running times.

\subsubsection{Theoretical Vs. Numerical Contraction Coefficients}

In this section, we compare theoretical contraction estimates against numerically computed values. Based on the parameters given in Table 6, the theoretical contraction estimate, as given in Theorem

3.3 , is computed as: $\left(\frac{M \alpha^{2}}{2 \lambda+2 M \lambda c_{f} \varphi_{0}+M \alpha^{2}}\right)^{2}=0.0003665$. By computing the ratio of the quantity of 


\begin{tabular}{|c|c|}
\hline Wells: & 3 production wells, 6 injection well \\
\hline Injection well (1): & Pressure specified, $4000.0 \mathrm{psi}$ \\
\hline Injection well (2): & Pressure specified, $3300.0 \mathrm{psi}$ \\
\hline Injection well (3): & Pressure specified, $4000.0 \mathrm{psi}$ \\
\hline Injection well (4): & Pressure specified, $4400.0 \mathrm{psi}$ \\
\hline Injection well $(5)$ : & Pressure specified, $3700.0 \mathrm{psi}$ \\
\hline Injection well $(6)$ : & Pressure specified, $4400.0 \mathrm{psi}$ \\
\hline Production well (1): & Pressure specified, 2000.0 psi \\
\hline Production well (2): & Pressure specified, $2000.0 \mathrm{psi}$ \\
\hline Production well (3): & Pressure specified, 2000.0 psi \\
\hline Total Simulation time: & 480.0 days \\
\hline Finer (Unit) time step: & 1.0 days \\
\hline Number of grids: & 1428 grids $(34 \times 14 \times 3)$ \\
\hline Permeabilities: $k_{x x}, k_{y y}, k_{z z}$ & highly varying, range: $(5.27 \mathrm{E}-10,3.10 \mathrm{E}+3) \mathrm{md}$ \\
\hline Initial porosity, $\varphi_{0}$ & 0.2 \\
\hline Fluid viscosity, $\mu_{f}$ & $2.0 \mathrm{cp}$ \\
\hline Initial pressure, $p_{0}$ & 400.0 psi \\
\hline Fluid compressibility $c_{f}$ : & 1.E-4 (1/psi) \\
\hline Rock compressibility: & 1.E-6 (1/psi) \\
\hline Rock density: & $165.44 l b_{m} / \mathrm{ft}^{3}$ \\
\hline Initial fluid density, $\rho_{f}$ : & $56.0 l b_{m} / f t^{3}$ \\
\hline Young's Modulus (E) & $1.2 \mathrm{E} 6 \mathrm{psi}$ \\
\hline Possion Ratio, $\nu$ & 0.35 \\
\hline Biot's constant, $\alpha$ & 0.9 \\
\hline Biot Modulus, $M$ & $1.0 \mathrm{E} 8 \mathrm{psi}$ \\
\hline$\lambda=\frac{E \nu}{(1+\nu)(1-2 \nu)}$ & $1.037 \mathrm{E} 6 \mathrm{psi}$ \\
\hline$L$ (introduced fixed stress parameter) & $\frac{\alpha^{2}}{2 \lambda}$ \\
\hline Flow Boundary Conditions: & no flow boundary condition on all 6 boundaries \\
\hline Mechanics B.C.: & \\
\hline "X+" boundary $(\operatorname{EBCXX1())}$ & $\sigma_{x x}=\sigma \cdot n_{x}=10,000 p s i$, (overburden pressure \\
\hline "X-" - boundary (EBCXXN1()) & $\boldsymbol{u}=0$, zero displacement \\
\hline "Y+" - boundary (EBCYY1()) & $\boldsymbol{u}=0$, zero displacement \\
\hline "Y-" - boundary (EBCYYN1()) & $\sigma_{y y}=\sigma \cdot n_{y}=2000 \mathrm{psi}$ \\
\hline "Z+" - boundary $(\operatorname{EBCZZ1())}$ & $\boldsymbol{u}=0$, zero displacement \\
\hline "Z-" - boundary (EBCZZN1()) & $\sigma_{z z}=\sigma \cdot n_{z}=1000 p s i$ \\
\hline
\end{tabular}

Table 6: Input Parameters for Frio Field Model 

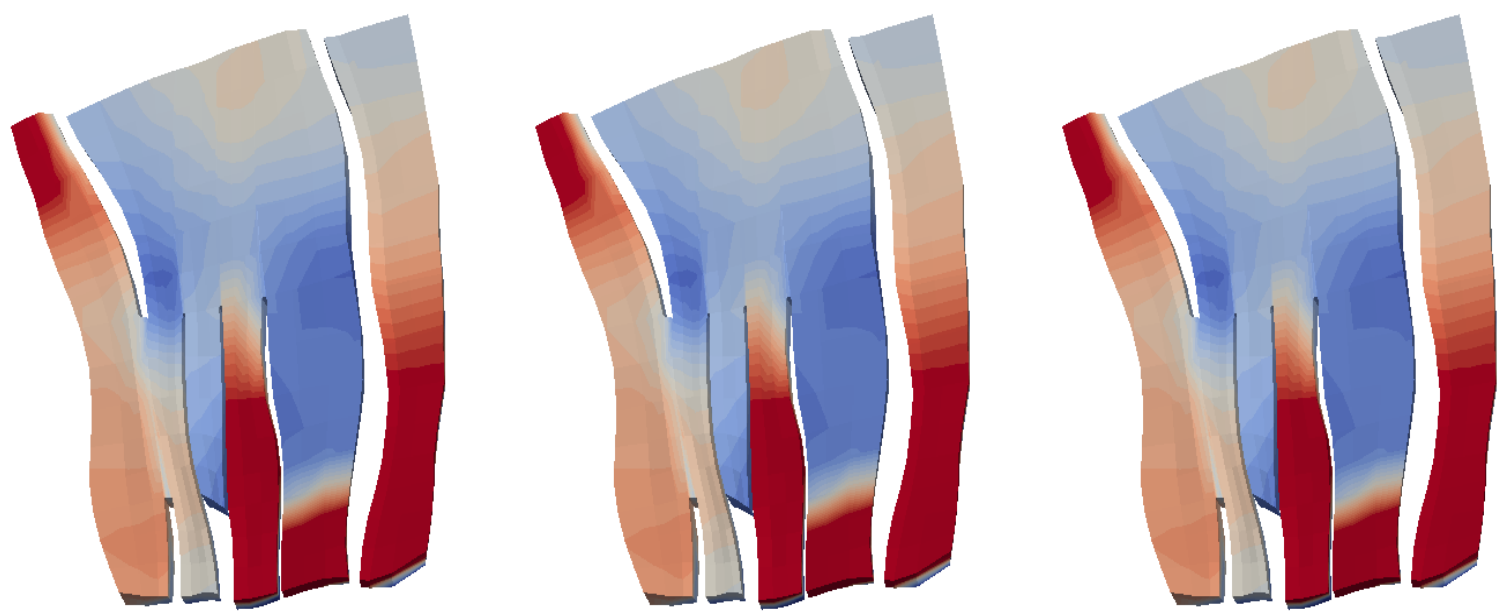

(a) Pressure Profiles after 480.0 simulation days

Disp. ( $q=1$ ) Magnitude

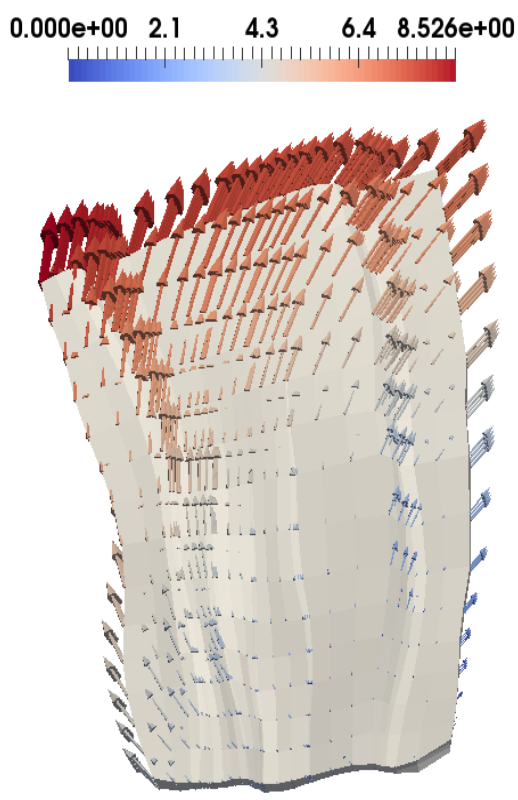

Disp. (q=4) Magnitude
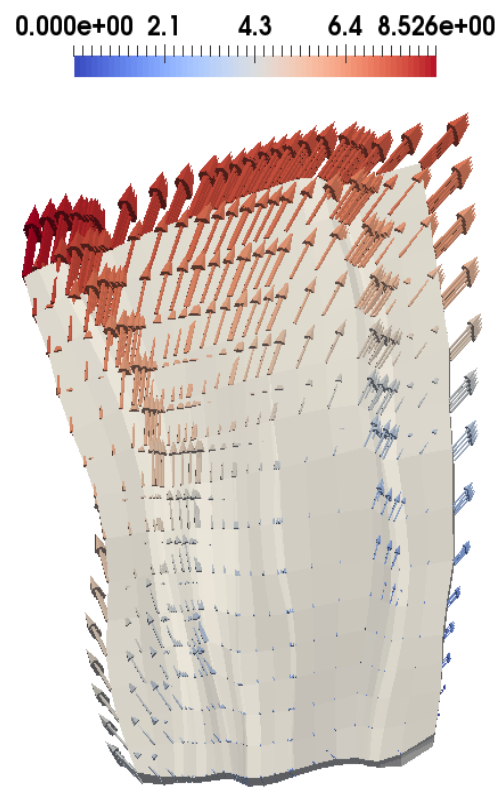

Disp. $(q=8)$ Magnitude

$\begin{array}{lllll}0.000 e+00 & 2.1 & 4.3 & 6.4 & 8.526 e+00\end{array}$

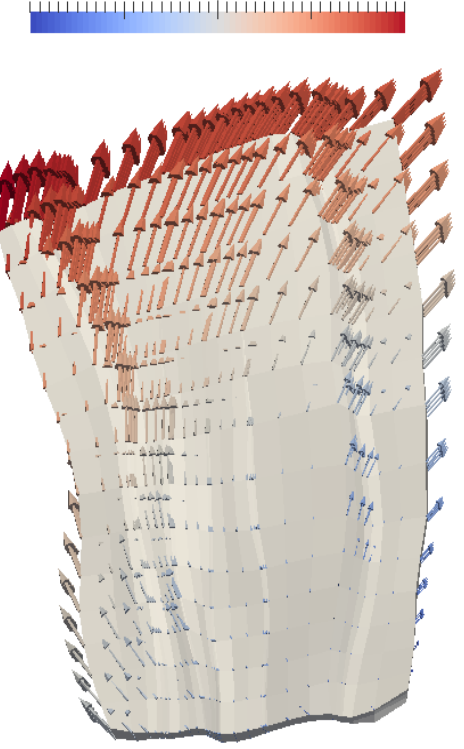

(b) Displacement Field after 480.0 simulation days

Figure 5.6: Frio Field Model Pressure and Displacement Fields at The End of The Simulation 


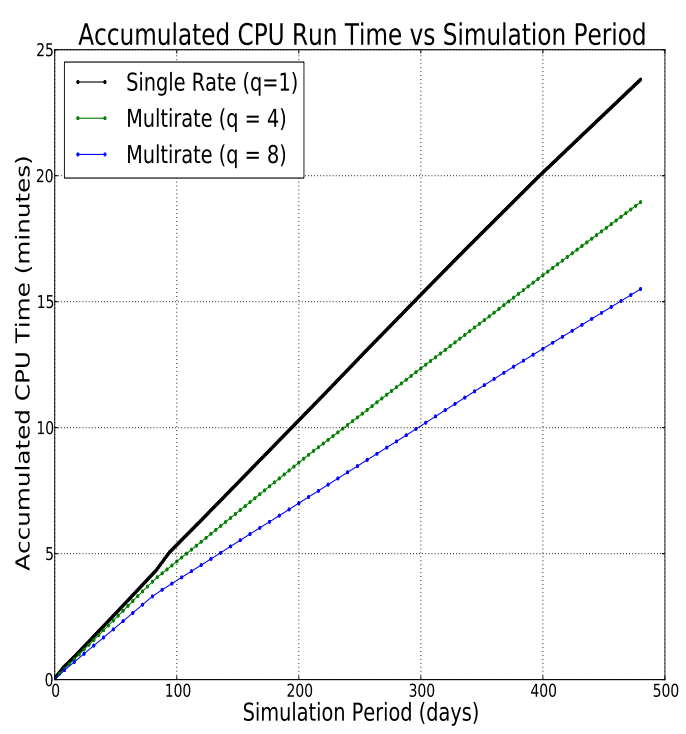

(a) CPU Run Time vs Simulation Days

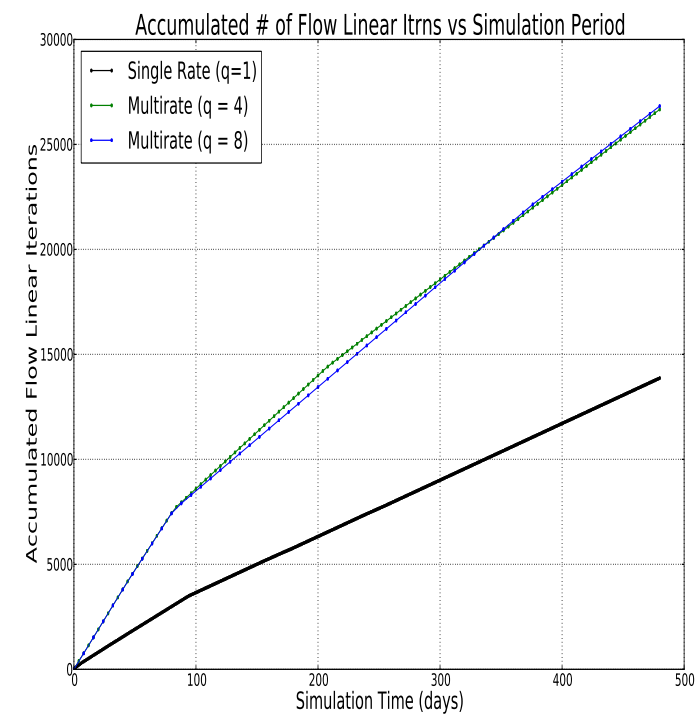

(c) Total Number of Flow Linear Iterations vs Simulation Days

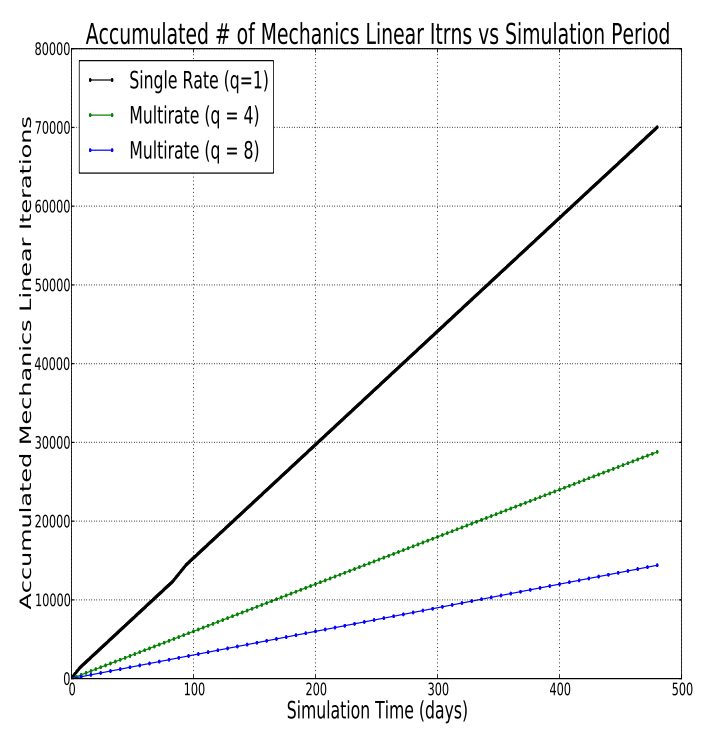

(b) Total Number of Mechanics Linear Iterations vs Simulation Days

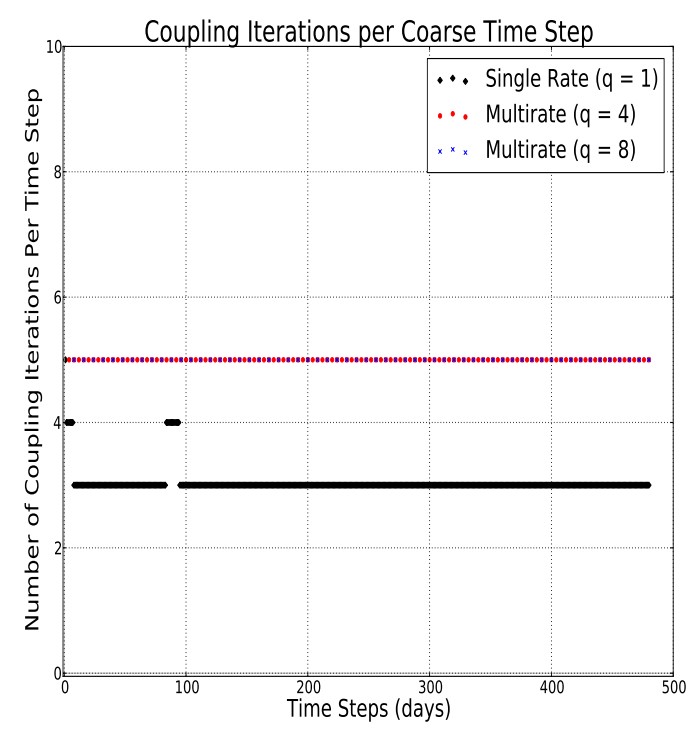

(d) Number of Iterative Coupling Iterations Per Coarser Time Step

Figure 5.7: Frio Field Model Simulation Results: CPU time savings of two multirate schemes $(q=4$ and 8$)$ over the single rate scheme $(q=1)$ are shown in the top left plot. The top right plot illustrates the huge reduction in the number of mechanics linear iterations for the corresponding multirate schemes over the single rate scheme. The bottom left plot illustrates the increase in the number of flow linear iterations for the multirate schemes. This is a direct consequence of the increase in the number of flow-mechanics coupling iterations observed for multirate schemes over the single rate scheme as shown in the bottom right plot. 
contraction between two consecutive iterative coupling iterations, numerical contraction estimates can be obtained. For the single rate case, this is computed as follows:

$$
\begin{aligned}
\left\|\delta \sigma_{v}^{n, k}\right\|_{\Omega}^{2} & =\left\|\delta p_{h}^{n, k}-\frac{\alpha}{L} \nabla \cdot \delta \boldsymbol{u}_{h}^{n, k}\right\|^{2} \\
& =\left.\frac{4 \lambda^{2}}{\alpha^{2}} \sum_{i, j, k}\left(\nabla \cdot \delta \boldsymbol{u}_{h}^{n, k}\right)^{2}\right|_{i, j, k} V(i, j, k)-\left.\left.\frac{4 \lambda}{\alpha} \sum_{i, j, k}\left(\nabla \cdot \delta \boldsymbol{u}_{h}^{n, k}\right)\right|_{i, j, k}\left(\delta p_{h}^{n, k}\right)\right|_{i, j, k} V(i, j, k) \\
& +\left.\sum_{i, j, k}\left(\delta p_{h}^{n, k}\right)^{2}\right|_{i, j, k} V(i, j, k)
\end{aligned}
$$

where $V(i, j, k)$ is the bulk volume of the $(i, j, k)$ grid block. Following this approach, the ratio of $\left(\left\|\delta \sigma_{v}^{n+1, m+k}\right\|_{/}^{2}\left\|\delta \sigma_{v}^{n, m+k}\right\|^{2}\right)$ for the single rate scheme is shown in figure 5.8. The maximum value, across all iterative coupling iterations, for each time step is plotted. Results illustrate that the theoretical estimate acts as an upper bound for numerically computed estimates. In addition, numerical contraction estimates are larger for earlier time steps. This is expected as the coupled problem has not reached the steady-state yet.

Figure 5.8: Numerical Contraction Estimates per Time Step. The maximum contraction estimate across flow-mechanics coupling iterations is considered for each time step

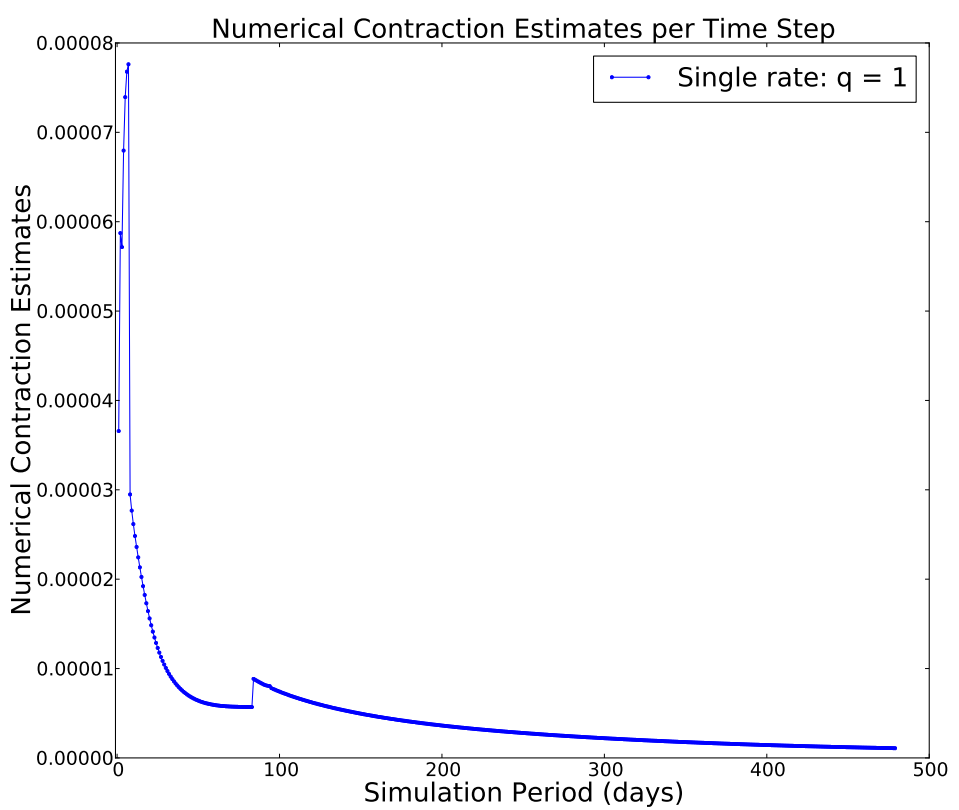


We identify three factors that determine the efficiency of multirate schemes: 1 . The relative computational cost of the flow solve versus the mechanics solve: if the computational cost of solving the coupled problem is dominated by the mechanics solve, then reducing the number of mechanics solve will substantially reduce the overall running time compared to single rate schemes. The multirate schemes are expected to be more useful in this case. 2. Longer simulation periods lead to larger time savings. During early time steps in the simulation, relatively larger numbers of coupling iterations are observed. As the model reaches mechanics equilibrium, the number of iterative coupling iterations per coarse mechanics time step gets reduced. This suggests a dynamic iterative coupling scheme, in which a single rate scheme is employed during early time steps in the simulation, and as the problem approaches mechanics equilibrium, multirate scheme should be employed with adaptive q. 3. Tolerance values used in the convergence stopping criteria affect the efficiency of multirate coupling schemes as well. Loose tolerance values reduce the number of iterative coupling iterations per coarse mechanics time step, which in turn reduces the overall running time. It is a tradeoff between the desired level of accuracy versus computational efficiency and is problem dependent.

Although the theory provided in this work and the numerical example are for single phase flow, we anticipate that multirate iterative coupling schemes will be of more importance for nonlinear flow problems coupled with geomechanics, as nonlinearities in the flow problem impose restrictions on the flow time step size. The multirate iterative coupling scheme would be a natural candidate for such nonlinear flow problems coupled with geomechanics.

\section{Conclusions and outlook}

We have considered two multirate iterative coupling schemes based on the fixed stress split iterative coupling algorithm. For both schemes, we have proved a Banach fixed-point contraction type estimate and convergence to the weak solution of the corresponding multirate fully discrete scheme. The first scheme is a natural extension of the single rate scheme, and contracts on a composite quantity consisting of pressure and volumetric strain terms. The second scheme exhibits the feature that it contracts on the same volumetric mean total stress defined in the single rate scheme. This is accomplished by successively correcting the fluxes during even coupling iterations. In addition, these flux corrections vanish as the coupling iteration approaches convergence. In contrast to the first multirate scheme, the modified multirate scheme has the same rate of convergence as in the single rate scheme.

Our analysis limits to one coarser time step and we have not studied the propagation of error due to temporal and spatial discretizations. These error estimates providing the convergence rate can be performed, for example, in the spirit of [16]. Further, the nonlinear extensions of these algorithms, their analyses and computational performance are interesting questions that will be addressed in

future work. Moreover, the performance of these algorithms should be further investigated to 
design coupling algorithms within the predefined tolerance with minimal computational costs. The extension to heterogeneous coefficients will be treated in our future work.

\section{Acknowledgements}

TA is funded by Saudi Aramco. We thank Paulo Zunino and Ivan Yotov for helpful discussions. KK would like to acknowledge the support of StatOil Akademia Grant (Bergen). The authors would like to acknowledge the CSM Industrial Affiliates program, DOE grant ER25617, and ConocoPhillips grant UTA10-000444.

\section{References}

[1] Y. Abousleiman, A. H.-D. Cheng, L. Cui, E. Detournay, and J.-C. Roegiers. Mandel's problem revisited. Géotechnique, 46(2):187-195, 1996.

[2] T. Almani, K. Kumar, G. Singh, and M. F. Wheeler. Stability of multirate explicit coupled of geomechanics with flow in a poroelastic medium. Ices report 16-12, Institute for Computational Engineering and Sciences, The University of Texas at Austin, Austin, Texas, 2016.

[3] T. Almani, K. Kumar, G. Singh, and M. F. Wheeler. Multirate undrained splitting for coupled flow and geomechanics in porous media. In ENUMATH 2015 Proceedings. European Conference on Numerical Mathematics and Advanced Applications, September 14-18, 2015. submitted.

[4] T. Almani, K. Kumar, and M. F. Wheeler. Multirate undrained splitting for coupled flow and geomechanics in porous media. Ices report 16-13, Institute for Computational Engineering and Sciences, The University of Texas at Austin, Austin, Texas, 2016.

[5] M. A. Biot. Consolidation settlement under a rectangular load distribution. J. Appl. Phys., 12(5):426-430, 1941.

[6] M. A. Biot. General theory of three-dimensional consolidation. J. Appl. Phys., 12(2):155-164, 1941.

[7] N. Castelletto, J. A. White, and H. A. Tchelepi. Accuracy and convergence properties of the fixed-stress iterative solution of two-way coupled poromechanics. International Journal for Numerical and Analytical Methods in Geomechanics, 2015.

[8] N. Castelletto, J. A. White, and H. A. Tchelepi. A unified framework for fully-implicit and sequential-implicit schemes for coupled poroelasticity. In ECMOR XIV. 14th European Conference on the Mathematics of Oil Recovery, Sep. 8-11, 2014. 
[9] L. Y. Chin, L. K. Thomas, J. E. Sylte, and R. G. Pierson. Iterative coupled analysis of geomechanics and fluid flow for rock compaction in reservoir simulation. Oil and Gas Science and Technology, 57(5):485-497, 2002.

[10] O. Coussy. A general theory of thermoporoelastoplasticity for saturated porous materials. Transport in Porous Media, 4:281-293, June 1989.

[11] X. Gai. A coupled geomechanics and reservoir flow model on parallel computers. PhD thesis, The University of Texas at Austin, Austin, Texas, 2004.

[12] X. Gai, R. H. Dean, M. F. Wheeler, and R. Liu. Coupled geomechanical and reservoir modeling on parallel computers. In The SPE Reservoir Simulation Symposium, Houston, Texas, Feb. 3-5, 2003.

[13] X. Gai, S. Sun, M. F. Wheeler, and H. Klie. A timestepping scheme for coupled reservoir flow and geomechanics on nonmatching grids. In SPE Annual Technical Conference and Exhibition, 2005. SPE97054.

[14] V. Girault, K. Kumar, and M. F. Wheeler. Convergence of iterative coupling of geomechanics with flow in a fractured poroelastic medium. Ices report 15-05, Institute for Computational Engineering and Sciences, The University of Texas at Austin, Austin, Texas, 2015.

[15] V. Girault, G Pencheva, M. F. Wheeler, and T. Wildey. Domain decomposition for poroelasticity and elasticity with dg jumps and mortars. Mathematical Models and Methods in Applied Sciences, 21(1):169-213, 2011.

[16] V. Girault, M. F. Wheeler, B. Ganis, and M. Mear. A lubrication fracture model in a poroelastic medium. Technical report, The Institute for Computational Engineering and Sciences, The University of Texas at Austin, 2013.

[17] M. Juntunen and M. F. Wheeler. Two-phase flow in complicated geometries - modeling the frio data using improved computational meshes. Computational Geosciences, 17:239-247, 2013.

[18] J. Kim, H. A. Tchelepi, and R. Juanes. Stability and convergence of sequential methods for coupled flow and geomechanics: fixed-stress and fixed-strain splits. Comput. Methods Appl. Mech. Engrg., 200(13-16):1591-1606, 2011.

[19] J. Kim, H. A. Tchelepi, and R. Juanes. Stability, accuracy, and efficiency of sequential methods for coupled flow and geomechanics. In The SPE Reservoir Simulation Symposium, Houston, Texas, February 2-4, 2009. SPE119084.

[20] R. A. Klausen, F. A. Radu, and G. T. Eigestad. Convergence of mpfa on triangulations and for richards' equation. International Journal for Numerical Methods in Fluids, 58(12):1327-1351, 2008 . 
[21] Runhild A. Klausen and Ragnar Winther. Convergence of multipoint flux approximations on quadrilateral grids. Numerical Methods for Partial Differential Equations, 22(6):1438-1454, 2006 .

[22] M. Mainguy and P. Longuemare. Coupling fluid flow and rock mechanics: formulations of the partial coupling between reservoir and geomechanics simulators. Oil and Gas Science and Technology - Rev. IFP, 57(4):355-367, 2002.

[23] J. Mandel. Consolidation des sols (Étude mathématique). Géotechnique, 3(7):287-299, 1953.

[24] A. Mikelić, B. Wang, and M. F. Wheeler. Numerical convergence study of iterative coupling for coupled flow and geomechanics. Computational Geosciences, 18:325-341, 2014.

[25] A. Mikelić and M. F. Wheeler. Convergence of iterative coupling for coupled flow and geomechanics. Computational Geosciences, 17:455-461, 2013.

[26] P. J. Phillips and M. F. Wheeler. A coupling of mixed and continuous Galerkin finite element methods for poroelasticity. I. The continuous in time case. Comput. Geosci., 11(2):131-144, 2007.

[27] Phillip Joseph Phillips and Mary F. Wheeler. A coupling of mixed and continuous galerkin finite element methods for poroelasticity i: the continuous in time case. Computational Geosciences, 11(2):131-144, 2007.

[28] A. Settari and F. Mourits. A coupled reservoir and geomechanical simulation system. SPE Journal, Sep. 1998.

[29] A. Settari and F. M. Mourits. Coupling of geomechanics and reservoir simulation models. In Siriwardane and Zema, editors, Comp. Methods and Advances in Geomech., pages 2151-2158, Balkema, Rotterdam, 1994.

[30] Li Shan, Haibiao Zheng, and William J. Layton. A decoupling method with different subdomain time steps for the nonstationary stokes?darcy model. Numerical Methods for Partial Differential Equations, 29(2):549-583, 2013.

[31] R. E. Showalter. Diffusion in poro-elastic media. J. Math. Anal. Appl., 251(1):310-340, 2000.

[32] G. Singh. Coupled Flow and Geomechanics Modeling for Fractured Poroelastic Reservoirs. PhD thesis, The University of Texas at Austin, Austin, Texas, 2014.

[33] K. von Terzaghi. Theoretical Soil Mechanics. Wiley, New York, 1943.

[34] B. Wang. Parallel Simulation of Coupled Flow and Geomechanics in Porous Media. PhD thesis, The University of Texas at Austin, Austin, Texas, 2014. 
[35] M. F. Wheeler, G. Xue, and I. Yotov. A family of multipoint flux mixed finite element methods for elliptic problems on general grids. In Procedia Computer Science, volume 4, pages 918-927. International Conference on Computational Science, ICCS 2011, 2011.

[36] M. F. Wheeler and I. Yotov. A multipoint flux mixed finite element method. SIAM Journal of Numerical Analysis, 44:2082-2106, 2006.

[37] Mary Wheeler, Guangri Xue, and Ivan Yotov. A multipoint flux mixed finite element method on distorted quadrilaterals and hexahedra. Numerische Mathematik, 121(1):165-204, 2012.

[38] X. Xiong. Analysis of a multi-rate splitting method for uncoupling evolutionary groundwatersurfacewater flows. Technical report, University of Pittsburgh, Aug. 8, 2012. 